\title{
51st ANNUAL SASKATCHEWAN CHRISTMAS BIRD COUNT - 1992
}

Compiled by WAYNE C. HARRIS, Box 414, Raymore, Saskatchewan. SOA $3 \mathrm{JO}$

Although the number of counts is down $20 \%$ to 80 from last year's record 99 counts, it is typical of the average for the past five years. In last year's compilation I requested $-50^{\circ} \mathrm{C}$ with strong winds for future counts and gratefully my request was granted. The temperature plummeted from unseasonably warm temperatures in the precount period to $-30^{\circ}$ to $-50^{\circ} \mathrm{C}$ for the entire count period and this was accompanied by strong winds on most days and as anticipated fewer people participated in counts, fewer counts were received, fewer species and numbers of individuals were reported.

\section{Weather and Coverage Table 1} summarizes the weather conditions encountered on the counts. Most temperatures were in the $-40^{\circ}$ to $-20^{\circ} \mathrm{C}$ range with but one plus temperature recorded for the entire count period. Winds were generally quite strong and combined with the low temperatures meant bitterly cold conditions for most of the counts. The most dramatic change in weather occurred at Leader $31 \mathrm{De}$ cember when the temperature rose from $-40^{\circ}$ to $0^{\circ} \mathrm{C}$ during the count.

In spite of the cold conditions 528 observers turned out for the counts and they tallied 994 hours observing birds (see Count areas and Participants and Table 2). All of these are down from last year. Table 2 also summarizes the habitats covered on each of the counts.
The Birds There were 77 species reported on count day with an additional 7 species seen during the count period but not found on count day. Just over 61,000 individuals were reported, down about $18 \%$ from last year and $39 \%$ from the record 1991 numbers. Many people commented on the lack of birds and the figures certainly confirm these comments. Table 7 changes in numbers when 1992 results are compared to 1991 and to an average of the five year period from 1987 through 1991. The lack of winter finches was most notable with redpolls, crossbills and Pine Grosbeaks being scarce. Red Crossbills were absent from the count for the first time in at least 20 years. Even the most abundant birds such as magpies, Blue Jays, Ruffed Grouse, Sharp-tailed Grouse and Gray Partridge decreased substantially.

Saskatoon again led the way with the greatest variety of species with 34 , compared to 42 last year. Fort Qu'Appelle and Regina were the only other counts to exceed 30 species, both with 32 . The average number of species per count was a meager 14 count day and only 16 for count period.

The most frequently occurring species was again the Black-billed Magpie (76 of 80 counts), followed by Black-capped Chickadee (71) and House Sparrow (67). The most abundant bird was the House Sparrow 
$(14,277$ individuals) followed closely by the Snow Bunting $(13,134)$. Tables 3,4 and 5 provide details of the 1992 counts.

New Species In spite of the extreme conditions two new species were added to the all time list bringing the total to 160 species. The first new species was a Lincoln's Sparrow recorded as a count period species at Whitewood. This individual had been present at a feeder up until the cold weather hit and then disappeared 18 December.

The second new species was the House Finch reported from both Regina and Weyburn. The Regina birds were found on count day but at Weyburn they were reported only as additional species for count period. The House Finch has been expanding its range throughout North America and it appeared only to be a matter of time before they were found in Saskatchewan during the winter.

Rare Species Several species though not new do warrant mention. A Yellow-billed Loon was present at Gardiner Dam for the second year in a row and like last year's record was an immature which would seem to preclude this being the same individual. A count period Great Blue Heron at Crooked Lake was only the third count record. A count period Varied Thrush at Swift Current was only the eighth count record. Several new high counts were recorded and these are found in Table 6.

Count Areas and Participants (Names of compilers are in italics. Number of participants in each count are in parentheses following the names.)

1. ABERNETHY-KATEPWA LAKE.
Ron Bittner, Ron Hooper, Joy McKen, Norm McKen, Richard Price, Keith Stephens, Wanda Steuk. (7)

2. ARMIT. Valeri Harris, Wayne Harris. (2)

3. BETHUNE. Doug and Vera Laing. (2)

4. BIGGAR. Mary-Anne Assailly, Roger Assailly, Penny Dyck, Greg Goring, Art Schmirler, Shirley Schmirler, Guy Wapple, Robert Wapple, Sandra Wapple. (9)

5. BIG MUDDY LAKE. Martin Bailey, Carol Bjorklund, Jill Forrester, Wayne Harris, Sheila Lamont. (5)

6. BIRCH HILLS. Marg Mareschal, Moe Mareschal. (2)

7. BROMHEAD. Martin Bailey, Carol Bjorklund. (2)

8. CABRI. Carman Dodge. (1)

9. CANDLE LAKE. Burke Korol, Harv Lane. (2)

10. CLARK'S CROSSING. Muriel Carlson, Bernie Gollop, Al Hartley, Menno Nickel, Ole Nielsen, Elsie Rayner, Stan Shadick, Wayne Shiels, Hilda Voth, Jim Wedgwood, Bob Whatley, Kay Whatley, Michael Williams, Lois Wooding. (14)

11. CRAVEN. Martin Bailey, Margaret Belcher, Al Binney, Betty Binnie, Carol Bjorklund, Trevor Herriot, Phyllis Isley, Bob Kreba, Ferne Lawrence, Jack McKenzie, Ron Myers, Frank Switzer. (12)

12. CROOKED LAKE. Dale Hjertaas, Estelle Hjertaas, Kerry Hjertaas, Warren Hjertaas, Bill Livsay, Martha Livsay, Dorothy Skene, Ed Skene. (8)

13. DILKE. Gary Barss, Margaret Belcher, Ken McArton, Bertha Wilson. (4)

14. DUVAL. George Herber, Linda Korytko, lain Richardson, Lloyd Saul. (4) 
15. EDAM. Marg and Tom Terpstra. (2)

16. ENDEAVOUR. Norman Harris. (1)

17. ESTEVAN. Guy Wapple. (1)

18. FENTON. Carman Dodge. (1)

19. FIFE LAKE. Martin Myers, Robert Rafuse. (2)

20. FORT QU'APPELLE. John Banick, Phyllis Bordass, William Bordass, Errol Cochrane, Ethel Cockwill,, Anne Davies, Evelyn Flemming, Doug Harman, Ronald Hooper, Jared Kaye, Vic Lamontagne, Jim Larocque, Maurice Lindgren, Don McDougall, Jean McKenna, Alan Mlazgar, Dolores Palmer, Paul Paquin, Lorne Rowell, Lloyd Talbot. (16)

21. FORT WALSH. Anne Harris, Valeri Harris, Wayne Harris, Burke Korol, Sheila Lamont, Wilkes Parsonage, Brenda Pollock, John Pollock, Dan Sawatzky, Guy Wapple, Robert Wapple. (11)

22. GARDINER DAM. Anne Harris, Valeri Harris, Wayne Harris, Burke Korol, Sheila Lamont, Wilkes Parsonage, Brenda Pollock, John Pollock, Dan Sawatzky, Guy Wapple, Robert Wapple. (11)

23. GOOD SPIRIT LAKE. Bill Anaka, Joyce Anaka, Julia Wiwchar. (3)

24. GOVENLOCK. Anne Harris, Valeri Harris, Wayne Harris, Burke Korol, Sheila Lamont, Wilkes Parsonage, Brenda Pollock, John Pollock, Dan Sawatzky, Guy Wapple, Robert Wapple. (11)

25. GRAND CENTRE - PIERCELAND. Frank Roy, Heidi Roy, Jessica Roy, Kathy Roy, Ryan Roy. (5)

26. GRASSLANDS

NATIONAL

PARK. Anne Harris, Valeri Harris, Wayne Harris, Sheila Lamont. (4)

27. HUDSON BAY. Moe Alain, Yvonne Alain, Pat Beaulieu,
Gerry Coates, Gerry Shuberg, Gloria Stang, Wayne Thacker. (7) 28. HUMBOLDT. Ed Brockmeyer, Mike Volk. (2)

29. INDIAN HEAD. Jan Beattie, Denise Beaulieu, Margo Beaulieu, Paul Beaulieu, Vic Beaulieu, Angela Gehl, David Gehl, Gordon Howe, John Kort, Peter Kort, Annette Meier, Lorne Scott, Anne Willerth, Gordon Willerth, Garth Willoughby, Jackie Willoughby. (17)

30. KAMSACK. John Barisoff, George and Marj Bernard, Ages Betz, Mike and Sally Bishop, Mable Buceuk, Lindee Duwore's, Bert and Doris Franklin, Anita Klocko, Bill Koroluk, W.J.C. May, Helen Panchuk, Dallas, Kelsey and Riley Rezansoff, Isabel Ritchie, Elsie Severson, John Solmon, Elenor Sookocheff, Phillyas Ward, Ann and Gordon Wlasichuk. (24)

31. KELVINGTON. Chester Sloan, Dianne Sloan, Marguerite Sloan, Pat Finnie. (4)

32. KENASTON. Doug Beckie, Lawrence Beckie. (2)

33. KINDERSLEY. Gerry Essar, Michael Essar. (2)

34. KINLOCH. Don Forbes, Doreen Forbes, Alice Rodenberg, Wilf Rodenberg. (4)

35. KUTAWAGAN LAKE. Wayne Harris, Sheila Lamont. (2)

36. LARONGE. Jim Paul, Lorie Ann Paul. (2)

37. LAST MOUNTAIN LAKE N.W.A. Wayne Harris. (1)

38. LEADER. Daisy Meyers. (1)

39. LIVELONG. Sarah Pavka, Vicky Tollefson. (2)

40. LOON LAKE. Bruce Lyle. (1)

41. LOVE-TORCH RIVER. Bent Dalziel, Duke Dalziel, Joan Dalziel, Kari Dalziel, Nora Dalziel, Sara Dalziel, Betty Donovan, Bruce Donovan, Sean Donovan, Ernie Hall, Viola King, Conrad 
L'Heureux, Elene L'Heureux, Paul L'Heureux, Terry Lazaruk, Mildred Long, Bill Matthews, Lynn Matthews. (18)

42. LUSELAND. Bill Frey, Kim Finley, Estelle Martfeld, Travis Martfeld. (4)

43. MACDOWALL. Myron Barton, Val Drummond, Henry Neudorf. (3)

44. MARSDEN. Eileen Graham, Candi Schopfer, Lois Wooding. (3)

45. MEADOW LAKE. Denise Saraur, Ann Sequin, Randy Sequin, Robbie Sequin, Bob Wilson, George Wood, Gladys Wood. (7)

46. MELFORT. Frieda Markland. (1)

47. MELVILLE. Donna Alberts, Ruby Alberts, Bill Barmby, Marion MacLean, Ainslie Wotherspoon, Robbie Wotherspoon, Ross Wotherspoon. (6)

48. MISSINIPE. Kathleen Callin, Joesph Glass, Shirley Glass. (3)

49. MOOSE JAW. Andy and Vi Anderson, Edith Bell, Doug and Helen Brunsdon, Muriel Dormer, Al Gurnsey, Mary Hembroff, Eve King, Leith Knight, Connie and Hugh McIntyre, Ramsay Ross, Arnie, Henry, John and Rob Van Dorland, Ed and Grace Walker. (19)

50. MOOSE MOUNTAIN. Ray Belanger, Greg Bobbitt, Ross Douglas, Dick Gutfriend, Phil Layh, Keith Sakatch, Stewart Stairmand. (6)

51. NICOLLE FLATS.

Edmonds, Alan Smith. (2)

52. NIPAWIN. Vi Budd, Joyce Christiansen, Doug Pegg. (3)

53. PIKE LAKE. Muriel Carlson, Mary Gilliland, Bernie Gollop, Mike Gollop, Debbie Lashta, Kevin Moore, Menno Nickel, Frank Roy, Stan Shadick, George Tosh, Jim Wedgwood, Lois Wooding. (12)

54. PORCUPINE PLAIN. Les Baker, David Black, Frank Gabriel,
Donald Hooper, Keith Larwood, Pat Larwood. (6)

55. PRINCE ALBERT(A). Elsie Morton. (1)

56. PRINCE ALBERT(B). Carol Beck, Ron Beck, John Burt, Pam Burt, Carman Dodge, Keith Dodge, Joe Graumans, Tim Loran, Ted Snow. (9)

57. PRINCE ALBERT NATIONAL PARK. Bruce Attikin, Susan Carr, Dave Dalman, Mary Dalman, Pat Fargey, Mark Kochems, Gail Lancaster, Howard Lancaster, Bradley Muir. (9)

58. QU'APPELLE VALLEY DAM. Bob Plaster, Frank Roy, Stan Shadick, Michael Williams. (4)

59. RAYMORE. Anne Harris, Valeri Harris, Wayne Harris, Sheila Lamont. (4)

60. REGINA. Jessie Bailey, Martin Bailey, Margaret Belcher, Carol Bjorklund, Joanne Bonneville, Lionel Bonneville, Stephane Bonneville, Tanis Davey, Jill Forrester, Malcolm Hayes, Trevor Herriot, Dale Hjertaas, Paule Hjertaas, Phyllis Ilsley, Olaf Jensen, Bob Kreba, Linda Hoyer Langenbacher, Rick Morrel, Ron Meyers, John Pollock, Tom Riffel, Margaret Skeel, Frank Switzer, Ian Switzer. (24)

61. REGINA BEACH. Daryl Johannesen. (1)

62. ROUND LAKE. Doug Francis, Joe and Laura Grimeau, Olga McDonald, John Trembley. (5)

63. SASKATOON. Tony Allen, Juhachi Asai, Kengo Asai, Rika Asai, Sumiko Asai, Garth Besant, Joyce. Besant, Robert Besant, Ray Bisha, Alice Boehm, Tony Diamond, Ed Driver, Anne Dzus, Fran Eldridge, Melanie Elliott, Martin Gerard, Silvia Gerard, Colleen Gerwing, Marshall Gilliland, Mary Gilliland, Sean Gilliland, Bernie Gollop, Madeleine Gollop, Jerry Haigh, John Hanbidge, 
Darlene Hay, Jim Hay, David Houston, Margaret Houston, Mary Houston, Stuart Houston, Grev Jones, Louise Jones, Venta Kabzems, Burke Korol, Rob Lavender, Cathryn Miller, David Miller, Lisa Napier, Garth Nelson, Menno Nickel, Shelly Ord, Steve Ord, Edith Rickman, Jim Smart, Jennifer Taylor, Phil Taylor, Grace Verrall, Heather Wagg, Jim Wedgwood, Shirley Wedgwood, Gordon Wilkinson, Joyce Wilkinson, Michael Williams, Dan Zazalenchuk. (55)

64. SCOTT. Guy Wapple. (1)

65. SKULL CREEK. Mark and Moreena Bell, Frank Ballauf, Jim Bennetto, Ray Bennetto, Bob Eccleston, Dorothy Eccleston, Greeba Drever, Rueben Flaig, Phyllis Flaig, Doris Bircham, Ralph Bircham, Bob Mann, Betty Mann, Marjorie Mann Jr., Pete Robertshaw, Patty Robertshaw, George Schultze, Ray Shuller, Michelle Shuller, Bret Wagner, Correy Wasilow, Harvey Wasilow, Mark Wasilow, Wade Wasilow, Brad Wolfater, Robin Wolfater. (25)

66. SNOWDEN. Irene Hagel, Karen Hagel, Hans Lien, Violet Lien, Sharon Lindberg, Lloyd Saul, Harold Thompson, Irene Thompson. (8)

67. SOMME - NEELY LAKE. Sharon Birch, Dawn Billeter, Edwin Billeter, Shirley Billeter, Louise Butterfield, Donald Hooper, Margaret Hooper. (7)

68. SPALDING. Velma Spizawka, William Spizawka. (2)

69. SPINNEY HILL. Ed Driver, Phil Taylor. (2)
70. SPRING VALLEY. Allan Bogdan, Flossie Bogdan, Larry Bogdan, Nick Bogdan, Dean Goian. (5)

71. SPRUCE HOME. Sharon and Tom Dice. (2)

72. SQUAW RAPIDS. Bert Dalziel, Joan Dalziel, Anne Harris, Valeri Harris, Wayne Harris, Burke Korol, Sheila Lamont, Guy Wapple, Robert Wapple. (9)

73. SWIFT CURRENT. James Beattie, Meryl Campion, Sara Froese, Ron Jensen, Doris Thoreson, Lois Wall, Russ Wall, Kae Waters, John Weston, Pearl Weston. (10)

74. TISDALE. Joyce Mohr. (1)

75. TURTLE LAKE. E.M. Robinson. (1)

76. VAL MARIE. Merv Hey, Jacques Saquet. (2)

77. WEYBURN. Leo Belanger, Louise Belanger, Ray Belanger, Greg Bobbitt, Norman Flaaten, Dick Gutfriend, Harry Kapasianyk, Norma Kapasianyk, Grace Kurtz, Betty Layh, Phil Layh, Bob Luterbach, Nick Postey, Keith Sakatch, Stewart Stairmand. (15)

78. WHITE BEAR. Daryl Jordheim, Sig Jordheim. (2)

79. WHITEWOOD. Pat Connelly, Boyd Metzler, John Pollock. (3)

80. YORKTON. Art Griffith, Russell Griffith, Dale Hjertaas, Kerry Hjertaas, Warren Hjertaas, Kenn Kozak, Roslyn Kozak, Geoff Rushowick, Patrick Rushowick, Dorothy Skene, Edward Skene, Duane Theaker, Sarah Theaker, Harold Wilkinson, Wilma Wilkinson. (15) 
Table 1. COUNT WEATHER CONDITIONS ( $T^{\circ} \mathrm{C}$, Wind $\mathrm{kmph}$, Snow $\mathrm{cm}$ )

\begin{tabular}{|c|c|c|c|c|c|c|c|c|}
\hline LOCALTY & $\begin{array}{l}\text { Min. } \\
T^{\circ} \mathrm{C}\end{array}$ & $\begin{array}{l}\text { Max. } \\
T^{\circ} \mathrm{C}\end{array}$ & $\begin{array}{l}\text { Min. } \\
\text { Wind }\end{array}$ & $\begin{array}{l}\text { Max. } \\
\text { Wind }\end{array}$ & $\begin{array}{l}\text { Min. } \\
\text { Snow } \\
\end{array}$ & $\begin{array}{l}\text { Max. } \\
\text { Snow }\end{array}$ & Sky A.M. & Sky P.M. \\
\hline $\begin{array}{l}\text { 1. ABERNETHY-KATEPWA LAKE } \\
\text { 2. ARMIT } \\
\text { 3. BETHUNE } \\
\text { 4. BIGGAR } \\
\text { 5. BIG MUDOY LAKE }\end{array}$ & $\begin{array}{l}-33 \\
-24 \\
-18 \\
-46 \\
-18 \\
\end{array}$ & $\begin{array}{r}-22 \\
-15 \\
-16 \\
-35 \\
-4 \\
\end{array}$ & $\begin{array}{r}10 \\
30 \\
15 \\
5 \\
0 \\
\end{array}$ & $\begin{array}{r}15 \\
60 \\
20 \\
10 \\
8 \\
\end{array}$ & $\begin{array}{r}12 \\
25 \\
20 \\
15 \\
0 \\
\end{array}$ & $\begin{array}{l}15 \\
30 \\
30 \\
31 \\
10 \\
\end{array}$ & $\begin{array}{l}\text { mostly dear } \\
\text { overcast } \\
\text { overcast } \\
\text { dear } \\
\text { mosty dear } \\
\end{array}$ & $\begin{array}{l}\text { dear } \\
\text { mosty doudy } \\
\text { mosty dear } \\
\text { dear } \\
\text { dear } \\
\end{array}$ \\
\hline $\begin{array}{l}\text { 6. BIRCH HILLS } \\
\text { 7. BROMHEAD } \\
\text { 8. CABRI } \\
\text { 9. CANDLE LAKE } \\
\text { 10. CLARK'S CROSSING } \\
\end{array}$ & $\begin{array}{l}-29 \\
-23 \\
-24 \\
-15 \\
-30 \\
\end{array}$ & $\begin{array}{l}-28 \\
-12 \\
-20 \\
.11 \\
.23 \\
\end{array}$ & $\begin{array}{r}5 \\
15 \\
10 \\
0 \\
0 \\
\end{array}$ & $\begin{array}{r}5 \\
25 \\
30 \\
5 \\
19 \\
\end{array}$ & $\begin{array}{r}20 \\
0 \\
8 \\
20 \\
10 \\
\end{array}$ & $\begin{array}{l}30 \\
10 \\
10 \\
30 \\
25 \\
\end{array}$ & $\begin{array}{l}\text { dear } \\
\text { partly doudy } \\
\text { party doudy } \\
\text { dear } \\
\text { dear }\end{array}$ & $\begin{array}{l}\text { dear } \\
\text { partly doudy } \\
\text { partly doudy } \\
\text { mostly doudy, light snow } \\
\text { dear }\end{array}$ \\
\hline $\begin{array}{l}\text { 11. CRAVEN } \\
\text { 12. CROOKED LAKE } \\
\text { 13. DIUKE } \\
\text { 14. DUVAL } \\
\text { 15. EDAM }\end{array}$ & $\begin{array}{l}-28 \\
-23 \\
-33 \\
-29 \\
-44 \\
\end{array}$ & $\begin{array}{l}-22 \\
-17 \\
-32 \\
-26 \\
-30 \\
\end{array}$ & $\begin{array}{r}5 \\
5 \\
10 \\
0 \\
30 \\
\end{array}$ & $\begin{array}{r}20 \\
10 \\
15 \\
8 \\
35 \\
\end{array}$ & $\begin{array}{r}15 \\
2 \\
8 \\
10 \\
\end{array}$ & \begin{tabular}{l|}
20 \\
20 \\
40 \\
12 \\
\end{tabular} & $\begin{array}{l}\text { dear } \\
\text { overcast } \\
\text { overcast } \\
\text { overcast, mod. snow } \\
\text { mosty doudy, mod. snow }\end{array}$ & $\begin{array}{l}\text { dear } \\
\text { overcast } \\
\text { overcast, verylight snow } \\
\text { overcast, heavy snow } \\
\text { overcast, mosty doudy } \\
\end{array}$ \\
\hline $\begin{array}{l}\text { 16. ENDEAVOUR } \\
\text { 17. ESTEVAN } \\
\text { 18. FENTON } \\
\text { 19. FIFE LAKE } \\
\text { 20. FOFT OU'APPELLE }\end{array}$ & $\begin{array}{l}-29 \\
-11 \\
-21 \\
-20 \\
-22 \\
\end{array}$ & $\begin{array}{r}+3 \\
-19 \\
-15 \\
-18 \\
\end{array}$ & $\begin{array}{r}0 \\
20 \\
0 \\
3 \\
2 \\
\end{array}$ & $\begin{array}{r}5 \\
90 \\
10 \\
10 \\
5 \\
\end{array}$ & $\begin{array}{r}20 \\
0 \\
5 \\
0 \\
15 \\
\end{array}$ & $\begin{array}{l}25 \\
10 \\
15 \\
10\end{array}$ & $\begin{array}{l}\text { mosily doudy } \\
\text { mosty doudy } \\
\text { mosty dear }\end{array}$ & $\begin{array}{l}\text { partly doudy } \\
\text { overcast, light snow } \\
\text { mosty dear } \\
\text { mosty dear }\end{array}$ \\
\hline $\begin{array}{l}\text { 21. FORT WALSH } \\
\text { 22. GARDINER DAM } \\
\text { 23. GOOD SPIRIT LAKE } \\
\text { 24. GOVENLOCK } \\
\text { 15. GRAND CENTRE-PIERCELAND }\end{array}$ & $\begin{array}{r}-8 \\
-25 \\
-35 \\
-20 \\
-20 \\
\end{array}$ & $\begin{array}{r}-15 \\
-23 \\
-24 \\
-8 \\
-19 \\
\end{array}$ & $\begin{array}{r}0 \\
20 \\
5 \\
0 \\
14 \\
\end{array}$ & $\begin{array}{l}20 \\
40 \\
12 \\
20 \\
16 \\
\end{array}$ & \begin{tabular}{r|}
0 \\
10 \\
15 \\
2 \\
25 \\
\end{tabular} & $\begin{array}{l}30 \\
20 \\
28 \\
10 \\
40 \\
\end{array}$ & $\begin{array}{l}\text { mostly doudy } \\
\text { mostly doudy } \\
\text { dear } \\
\text { partly doudy } \\
\text { overcast, light snow }\end{array}$ & $\begin{array}{l}\text { mostly doudy, light snow } \\
\text { mosty doudy } \\
\text { mosty dear } \\
\text { party doudy } \\
\text { party doudy }\end{array}$ \\
\hline $\begin{array}{l}\text { 26. GRASSLANDS NATIONALPARK } \\
\text { 27. HUDSON BAY } \\
\text { 28. HUMBOLDT } \\
\text { 29. INDIAN HEAD } \\
\text { 30. KAMSACK }\end{array}$ & $\begin{array}{l}-10 \\
-15 \\
-20 \\
-30 \\
-42 \\
\end{array}$ & $\begin{array}{l}-5 \\
-20 \\
-16 \\
-25 \\
-30 \\
\end{array}$ & $\begin{array}{r}10 \\
25 \\
5 \\
10 \\
20 \\
\end{array}$ & $\begin{array}{l}70 \\
30 \\
10 \\
15 \\
30 \\
\end{array}$ & $\begin{array}{r}2 \\
35 \\
3 \\
25 \\
60 \\
\end{array}$ & $\begin{array}{r}10 \\
45 \\
8 \\
30 \\
90 \\
\end{array}$ & $\begin{array}{l}\text { moslly dear } \\
\text { mostly dear } \\
\text { overcast, light snow } \\
\text { mosty dear }\end{array}$ & $\begin{array}{l}\text { partly doudy } \\
\text { party doudy } \\
\text { overcast light snow } \\
\text { dear } \\
\text { mostly dear }\end{array}$ \\
\hline $\begin{array}{l}\text { 31. KELVINGTON } \\
\text { 32. KENASTON } \\
\text { 33. KINDERSLEY } \\
\text { 34. KINLOCH } \\
\text { 35. KUTAWAGAN LAKE }\end{array}$ & $\begin{array}{l}-17 \\
-24 \\
-31 \\
-18 \\
-24 \\
\end{array}$ & $\begin{array}{l}-15 \\
-18 \\
-20 \\
-15 \\
-17 \\
\end{array}$ & $\begin{array}{r}0 \\
12 \\
10 \\
10 \\
10\end{array}$ & $\begin{array}{l}35 \\
20 \\
15 \\
20 \\
20 \\
\end{array}$ & $\begin{array}{l}50 \\
15 \\
10 \\
26 \\
20 \\
\end{array}$ & $\begin{array}{l}60 \\
20 \\
30 \\
36 \\
25 \\
\end{array}$ & $\begin{array}{l}\text { partly doudy } \\
\text { overcast, mod. snow } \\
\text { mosty dear } \\
\text { mosty dear }\end{array}$ & $\begin{array}{l}\text { mosty doudy } \\
\text { overcast, light snow } \\
\text { mostly dear } \\
\text { mosty doudy } \\
\text { mosty dear }\end{array}$ \\
\hline $\begin{array}{l}\text { 36. LARONGE } \\
\text { 37. LAST MOUNT AIN LAKE N.W.A. } \\
\text { 38. LEADER } \\
\text { 39. LVELONG } \\
\text { 40. LOON LAKE }\end{array}$ & $\begin{array}{l}-23 \\
-28 \\
-40 \\
-25 \\
-40 \\
\end{array}$ & \begin{tabular}{r|}
-15 \\
-20 \\
0 \\
-19 \\
-27 \\
\end{tabular} & $\begin{array}{r}7 \\
20 \\
0 \\
10 \\
0 \\
\end{array}$ & $\begin{array}{r}12 \\
70 \\
5 \\
15\end{array}$ & $\begin{array}{r}30 \\
5 \\
5 \\
25 \\
27 \\
\end{array}$ & $\begin{array}{l}50 \\
20 \\
30 \\
30\end{array}$ & $\begin{array}{l}\text { partly doudy } \\
\text { mostly doudy } \\
\text { mosty dear } \\
\text { mosty dear } \\
\text { dear } \\
\end{array}$ & $\begin{array}{l}\text { mostly doudy } \\
\text { overcast, mod. snow } \\
\text { dear } \\
\text { dear } \\
\text { mosty dear }\end{array}$ \\
\hline $\begin{array}{l}\text { 41. LOVE-TORCH RIVER } \\
\text { 42. LUSELAND } \\
\text { 43. MACDOWALL } \\
\text { 44. MARSDEN } \\
\text { 45. MEADOW LAKE }\end{array}$ & $\begin{array}{l}-40 \\
-34 \\
-30 \\
-47 \\
-33 \\
\end{array}$ & $\begin{array}{l}-33 \\
-30 \\
-25 \\
-23 \\
-30 \\
\end{array}$ & $\begin{array}{r}0 \\
5 \\
2 \\
0 \\
10 \\
\end{array}$ & $\begin{array}{r}5 \\
10 \\
6 \\
70 \\
40 \\
\end{array}$ & $\begin{array}{l}30 \\
10 \\
12 \\
10 \\
15 \\
\end{array}$ & $\begin{array}{l}45 \\
25 \\
18 \\
25 \\
75 \\
\end{array}$ & $\begin{array}{l}\text { mosity doudy } \\
\text { mosity dear } \\
\text { dear }\end{array}$ & $\begin{array}{l}\text { mosily doudy } \\
\text { mostly dear } \\
\text { dear }\end{array}$ \\
\hline $\begin{array}{l}\text { 46. MELFORT } \\
\text { 47. MELVILLE } \\
\text { 48. MISSINIPE } \\
\text { 49. MOOSE JAW } \\
\text { 50. MOOSE MOUNTAIN }\end{array}$ & $\begin{array}{l}-16 \\
-30 \\
-22 \\
.10 \\
-26 \\
\end{array}$ & $\begin{array}{r}-40 \\
-24 \\
-13 \\
.9 \\
-18 \\
\end{array}$ & $\begin{array}{r}10 \\
0 \\
18 \\
5 \\
\end{array}$ & 15 & $\begin{array}{r}5 \\
25 \\
12 \\
2 \\
\end{array}$ & $\begin{array}{l}30 \\
50 \\
10\end{array}$ & $\begin{array}{l}\text { mosty dear } \\
\text { mosty dear } \\
\text { dear } \\
\text { overcast } \\
\text { overcast }\end{array}$ & $\begin{array}{l}\text { mosty y dear } \\
\text { mosty y dear } \\
\text { dear } \\
\text { overcast } \\
\text { mosty y doudy }\end{array}$ \\
\hline $\begin{array}{l}\text { 51. NICOULE FLATS } \\
\text { 52. NIPAWIN } \\
\text { 53. PIKE LAKE } \\
\text { 54. PORCUPINE PLAIN } \\
\text { 55. PRINCE ALBERT (A) }\end{array}$ & \begin{tabular}{l|}
-20 \\
-14 \\
-22 \\
-35 \\
-32 \\
\end{tabular} & \begin{tabular}{l|}
-15 \\
-14 \\
.19 \\
.30
\end{tabular} & \begin{tabular}{r|}
0 \\
0 \\
15 \\
0
\end{tabular} & \begin{tabular}{r|}
15 \\
5 \\
17 \\
0
\end{tabular} & \begin{tabular}{r|}
10 \\
23 \\
5 \\
30
\end{tabular} & $\begin{array}{l}20 \\
61 \\
10 \\
35\end{array}$ & $\begin{array}{l}\text { overcast } \\
\text { dear }\end{array}$ & $\begin{array}{l}\text { overcast } \\
\text { mosjy clear }\end{array}$ \\
\hline $\begin{array}{l}\text { 56. PRINCE ALBERT (B) } \\
\text { 57. PRINCE ALBERT NAT, PARK } \\
\text { 58. OU'APPELLE VALLYY DAM } \\
\text { 59. RAYMORE } \\
\text { 60. REGINA }\end{array}$ & $\begin{array}{r}-36 \\
-31 \\
-20 \\
-24 \\
-18 \\
\end{array}$ & $\begin{array}{l}-30 \\
-28 \\
-17 \\
-18 \\
-12 \\
\end{array}$ & $\begin{array}{r}5 \\
10 \\
15 \\
0 \\
15 \\
\end{array}$ & $\begin{array}{l}20 \\
25 \\
20 \\
10 \\
40 \\
\end{array}$ & $\begin{array}{r}1 \\
25 \\
2 \\
15 \\
0 \\
\end{array}$ & $\begin{array}{l}10 \\
45 \\
12 \\
20 \\
10 \\
\end{array}$ & $\begin{array}{l}\text { mostly dear } \\
\text { dear } \\
\text { mosty doudy } \\
\text { mostly dear } \\
\text { moderate blowing snow }\end{array}$ & $\begin{array}{l}\text { mosty dear } \\
\text { dear } \\
\text { mosty doudy, light snow } \\
\text { mosty dear } \\
\text { moderate snow }\end{array}$ \\
\hline $\begin{array}{l}\text { 61. REGINA BEACH } \\
\text { 62. ROUND LAKE } \\
\text { 63. SASKATOON } \\
\text { 64. SCOTT } \\
\text { 65. SKULL CREEK }\end{array}$ & $\begin{array}{l}-20 \\
-25 \\
-22 \\
-20 \\
-22\end{array}$ & $\begin{array}{l}-16 \\
-15 \\
-20 \\
\end{array}$ & $\begin{array}{r}10 \\
10 \\
24 \\
5 \\
5\end{array}$ & $\begin{array}{l}15 \\
15 \\
37 \\
20 \\
10\end{array}$ & $\begin{array}{l}15 \\
30 \\
10 \\
10 \\
10\end{array}$ & $\begin{array}{l}40 \\
13 \\
30 \\
15\end{array}$ & $\begin{array}{l}\text { mostly doudy } \\
\text { mostly doudy, mod. snow } \\
\text { overcast, light snow \& fog } \\
\text { pty. doudy, It. snow \& log }\end{array}$ & $\begin{array}{l}\text { mostly doudy } \\
\text { mostly doudy, hvy. snow } \\
\text { overcast light snow } \\
\text { mostly ddy., It.snow \& fog }\end{array}$ \\
\hline
\end{tabular}


Table 1. COUNT WEATHER CONDITIONS (continued) $\left(T^{\circ} \mathrm{C}\right.$, Wind $\mathrm{kmph}$, Snow $\mathrm{cm}$ )

\begin{tabular}{|c|c|c|c|c|c|c|c|c|}
\hline LOCAUTYY & $\begin{array}{l}\text { Min. } \\
\mathrm{T}^{\circ} \mathrm{C} \\
\end{array}$ & $\begin{array}{l}\text { Max. } \\
T^{\circ} \mathrm{C}\end{array}$ & $\begin{array}{l}\text { Min. } \\
\text { Wind }\end{array}$ & $\begin{array}{l}\text { Max. } \\
\text { Wind }\end{array}$ & $\begin{array}{c}\text { Min. } \\
\text { Snow }\end{array}$ & $\begin{array}{l}\text { Max. } \\
\text { Snow }\end{array}$ & Sky A.M. & Sky P.M. \\
\hline $\begin{array}{l}\text { 66. SNOWDEN } \\
\text { 67. SOMME-NEELY LAKE } \\
\text { 68. SPALDING } \\
\text { 69. SPINNEY HILL } \\
\text { 70. SPRING VALLEY } \\
\end{array}$ & $\begin{array}{l}-22 \\
-20 \\
-22 \\
-21 \\
-26 \\
\end{array}$ & $\begin{array}{l}-21 \\
-10 \\
.15 \\
-21 \\
.19 \\
\end{array}$ & $\begin{array}{r}0 \\
0 \\
0 \\
0 \\
10 \\
\end{array}$ & $\begin{array}{r}15 \\
5 \\
5 \\
20 \\
15 \\
\end{array}$ & $\begin{array}{r}2 \\
30 \\
45 \\
2 \\
15 \\
\end{array}$ & $\begin{array}{l}30 \\
35 \\
60 \\
50 \\
18 \\
\end{array}$ & $\begin{array}{l}\text { dear } \\
\text { party doudy } \\
\text { party doudy } \\
\text { mostly dear } \\
\text { mostly doudy }\end{array}$ & $\begin{array}{l}\text { dear } \\
\text { pardy doudy } \\
\text { party doudy } \\
\text { pty. doudy, very lt. snow } \\
\text { mostly doudy }\end{array}$ \\
\hline $\begin{array}{l}\text { 71. SPRUCE HOME } \\
\text { 72. SQUAW RAPIDS } \\
\text { 73. SWFT CURRENT } \\
\text { 74. TISDALE } \\
\text { 75. TURTLE LAKE } \\
\end{array}$ & $\begin{array}{l}-33 \\
-20 \\
-21 \\
-30 \\
-35 \\
\end{array}$ & $\begin{array}{l}-25 \\
-11 \\
-15 \\
-25 \\
25 \\
\end{array}$ & $\begin{array}{r}5 \\
5 \\
20 \\
10 \\
5 \\
\end{array}$ & $\begin{array}{l}10 \\
15 \\
37 \\
20 \\
10 \\
\end{array}$ & $\begin{array}{r}25 \\
15 \\
2 \\
15 \\
30 \\
\end{array}$ & $\begin{array}{l}31 \\
20 \\
30 \\
20 \\
40 \\
\end{array}$ & $\begin{array}{l}\text { party doudy } \\
\text { mostly doudy } \\
\text { mostly doudy } \\
\text { mostly dear } \\
\text { dear }\end{array}$ & $\begin{array}{l}\text { pardy doudy } \\
\text { mostly doudy } \\
\text { mostly doudy } \\
\text { party doudy } \\
\text { dear }\end{array}$ \\
\hline $\begin{array}{l}\text { 76. VAL MARIE } \\
\text { 77. WEYBURN } \\
\text { 78. WHITE BEAR } \\
\text { 79. WHITEWOOD } \\
\text { 80. YORKTON }\end{array}$ & $\begin{array}{l}-22 \\
-28 \\
-23 \\
-32 \\
-20\end{array}$ & $\begin{array}{l}-15 \\
-25 \\
-21 \\
-20 \\
-18\end{array}$ & $\begin{array}{l}5 \\
0 \\
5 \\
0 \\
0\end{array}$ & $\begin{array}{r}15 \\
0 \\
10 \\
15 \\
8\end{array}$ & $\begin{array}{r}10 \\
5 \\
10 \\
10 \\
15\end{array}$ & $\begin{array}{r}15 \\
7 \\
30 \\
40 \\
40\end{array}$ & $\begin{array}{l}\text { dear } \\
\text { mosty dear } \\
\text { overcast light snow } \\
\text { dear } \\
\text { overcast }\end{array}$ & $\begin{array}{l}\text { mostly dear } \\
\text { pardy doudy } \\
\text { dear } \\
\text { dear }\end{array}$ \\
\hline
\end{tabular}

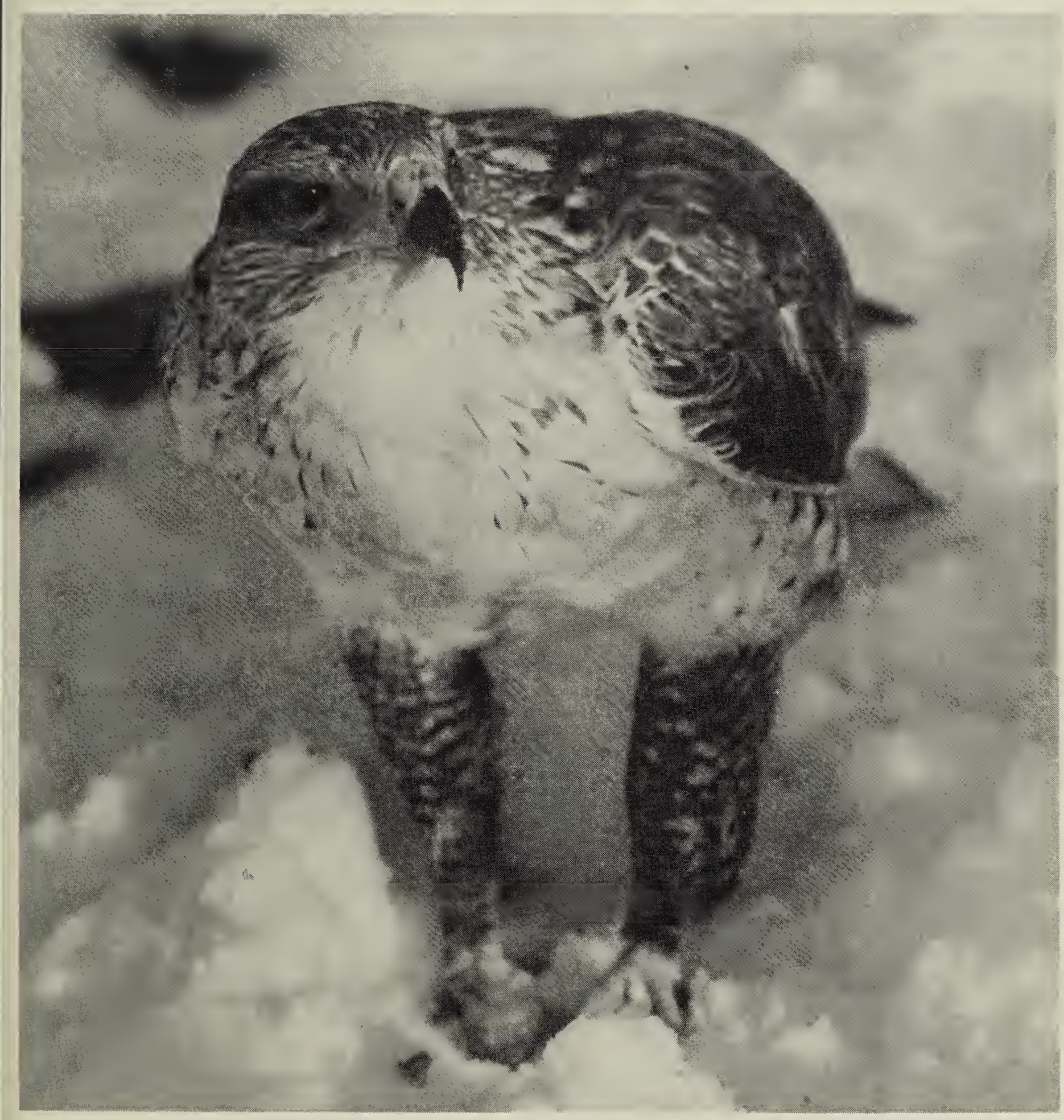

Ferruginous Hawk.

Wayne Lynch 
Table 2. COUNT COVERAGE ("indicates counts which conform to the North American standard of all day counts covering a 15-mile diameter circle)

\begin{tabular}{|c|c|c|c|c|c|c|c|c|c|c|c|c|c|c|c|c|}
\hline \multirow[b]{2}{*}{ COUNT NAME } & \multirow[b]{2}{*}{$\begin{array}{l}\overline{0} \\
\stackrel{0}{0} \\
\delta \\
\xi \\
\text { ริ }\end{array}$} & \multirow[b]{2}{*}{ 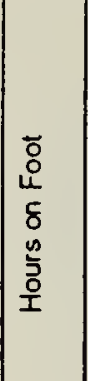 } & \multirow[b]{2}{*}{ 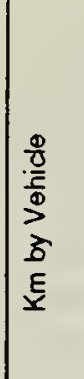 } & \multirow[b]{2}{*}{ 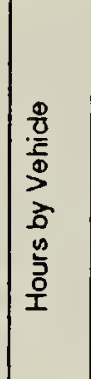 } & \multirow[b]{2}{*}{ 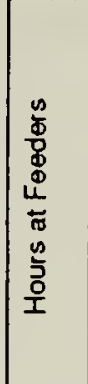 } & \multicolumn{11}{|c|}{ Habitat Covered (\%) } \\
\hline & & & & & & 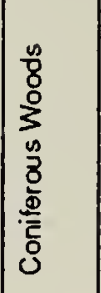 & $\begin{array}{l}\frac{n}{8} \\
\frac{0}{3} \\
\frac{3}{3} \\
0 \\
\frac{0}{2} \\
\frac{x}{2}\end{array}$ & $\begin{array}{l}0 \\
8 \\
8 \\
3 \\
0 \\
0 \\
0 \\
3 \\
0 \\
0 \\
0 \\
0\end{array}$ & 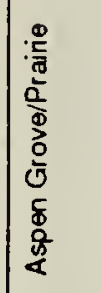 & 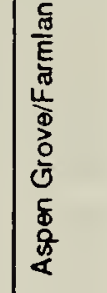 & 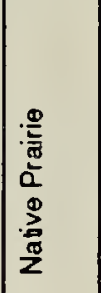 & 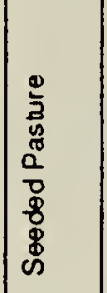 & 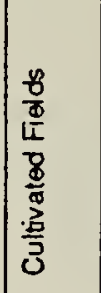 & 总 & $\frac{5}{8}$ & $\begin{array}{l}\frac{\Phi}{\pi / 0} \\
3 \\
\$ \\
\delta\end{array}$ \\
\hline $\begin{array}{l}\text { 1. ABERNETHY.KATEPWAL. } \\
\text { 2. ARMIT* } \\
\text { 3. BETHUNE } \\
\text { 4. BIGGAR* } \\
\text { 5. BIGMUDDY LAKE. }\end{array}$ & $\begin{array}{r}3 \\
10 \\
6\end{array}$ & $\begin{array}{r}3 \\
5 \\
2.5\end{array}$ & $\begin{array}{r}85 \\
106 \\
20 \\
193 \\
283\end{array}$ & $\begin{array}{r}3 \\
5 \\
3 \\
11.5 \\
11\end{array}$ & $\begin{array}{l}2 \\
1 \\
2\end{array}$ & $<5$ & $>75$ & $<5$ & & $\begin{array}{r}51.75 \\
6-25\end{array}$ & 6.25 & & $\begin{array}{r}6-25 \\
<5 \\
6-25 \\
51.75\end{array}$ & $\begin{array}{l}6-25 \\
6-25\end{array}$ & $\begin{array}{l}6-25 \\
6-25\end{array}$ & \\
\hline $\begin{array}{l}\text { 6. BIRCH HILLS } \\
\text { 7. BROMHEAD' } \\
\text { 8. CABRI } \\
\text { 9. CANDLE LAKE' } \\
\text { 10. CLARK'S CROSSING. }\end{array}$ & $\begin{array}{r}4 \\
5 \\
13 \\
\end{array}$ & $\begin{array}{r}1 \\
.5 \\
1.5 \\
13.9 \\
\end{array}$ & $\begin{array}{r}55 \\
195 \\
66.7 \\
145 \\
478 \\
\end{array}$ & $\begin{array}{r}3.5 \\
7 \\
2.5 \\
5 \\
17.8 \\
\end{array}$ & $\begin{array}{l}.5 \\
.5 \\
.5\end{array}$ & $6-25$ & $26-50$ & $\begin{array}{r}<5 \\
6-25 \\
<5 \\
\end{array}$ & $<5$ & $51-75$ & $6-25$ & $\begin{array}{r}<5 \\
6-25 \\
6-25 \\
\end{array}$ & $\begin{array}{r}51-75 \\
>75 \\
26-50 \\
\\
6-25 \\
\end{array}$ & $\begin{array}{r}6-25 \\
6-25 \\
6-25 \\
\end{array}$ & $\begin{array}{r}6-25 \\
<5 \\
<5 \\
\end{array}$ & $6-25$ \\
\hline $\begin{array}{l}\text { 11. CRAVEN } \\
\text { 12. CROOKEDLAKE } \\
\text { 13. DILKE } \\
\text { 14. DUVAL } \\
\text { 15. EDAM }\end{array}$ & $\begin{array}{r}14 \\
15 \\
2 \\
2\end{array}$ & $\begin{array}{r}9 \\
6 \\
.66 \\
1\end{array}$ & $\begin{array}{r}304 \\
70 \\
93 \\
85.5 \\
10 \\
\end{array}$ & $\begin{array}{r}14 \\
7 \\
5.33 \\
4 \\
1 \\
\end{array}$ & $\begin{array}{l}2 \\
1 \\
1 \\
4 \\
\end{array}$ & & & $26-50$ & $\begin{array}{r}26-50 \\
6-25\end{array}$ & $\begin{array}{r}6-25 \\
51-75\end{array}$ & $\begin{array}{r}<5 \\
6-25 \\
6-25\end{array}$ & & $\begin{array}{r}26-50 \\
\\
6-25 \\
26-50\end{array}$ & $\begin{array}{r}6-25 \\
<5 \\
6-25\end{array}$ & $\begin{array}{r}6-25 \\
<5 \\
6.25\end{array}$ & $<5$ \\
\hline $\begin{array}{l}\text { 16. ENDEAVOUR } \\
\text { 17. ESTEVAN' } \\
\text { 18. FENTON } \\
\text { 19. FIFE LAKE } \\
\text { 20. FORT OU'APPELLE }\end{array}$ & $\begin{array}{l}3 \\
6\end{array}$ & $\begin{array}{l}.5 \\
.5 \\
\end{array}$ & $\begin{array}{r}30 \\
80 \\
141 \\
150 \\
100 \\
\end{array}$ & $\begin{array}{r}2.5 \\
3.5 \\
2.75 \\
3 \\
7 \\
\end{array}$ & .5 & & & $26 \cdot 50$ & $\begin{array}{r}6-25 \\
6-25 \\
\end{array}$ & $\begin{array}{l}6-25 \\
6-25 \\
\end{array}$ & $<5$ & $\begin{array}{r}26-50 \\
6-25 \\
\end{array}$ & $\begin{array}{r}26-50 \\
26-50 \\
>75\end{array}$ & $\begin{array}{r}26-50 \\
6-25 \\
<5 \\
\\
6-25 \\
\end{array}$ & $\begin{array}{r}<5 \\
6-25 \\
6-25 \\
\end{array}$ & $\begin{array}{r}26-50 \\
<5 \\
6-25 \\
\end{array}$ \\
\hline $\begin{array}{l}\text { 21. FORT WALSH. } \\
\text { 22. GARDINER DAM* } \\
\text { 23. GOOD SPIRIT LAKE. } \\
\text { 24. GOVENLOCK } \\
\text { 25. GRD. CTR-PIERCELAND }\end{array}$ & $\begin{array}{r}74 \\
19 \\
3 \\
16 \\
2.5 \\
\end{array}$ & $\begin{array}{r}30 \\
10 \\
1 \\
8 \\
1.5 \\
\end{array}$ & $\begin{array}{l}177 \\
298 \\
105 \\
320 \\
121 \\
\end{array}$ & $\begin{array}{r}11 \\
14.5 \\
5 \\
13 \\
4 \\
\end{array}$ & 1 & $\begin{array}{l}<5 \\
<5 \\
\end{array}$ & $\begin{array}{r}26-50 \\
<5 \\
26-50 \\
\end{array}$ & $\begin{array}{l}6-25 \\
6-25 \\
6-25 \\
\end{array}$ & $\begin{array}{r}<5 \\
6-25\end{array}$ & 51.75 & $\begin{array}{r}<5 \\
<5 \\
51 \cdot 75\end{array}$ & $6-25$ & $\begin{array}{r}6-25 \\
26-50 \\
26-50 \\
26-50 \\
6-25 \\
\end{array}$ & \begin{tabular}{r|}
$26-50$ \\
$6-25$ \\
$6-25$ \\
$6-25$ \\
\end{tabular} & $\begin{array}{r}<5 \\
<5 \\
6-25 \\
\end{array}$ & $\begin{array}{r}26-50 \\
6-25\end{array}$ \\
\hline $\begin{array}{l}\text { 26. GRASSLANDSN.P: } \\
\text { 27. HUDSON BAY } \\
\text { 28. HUMBOLDT } \\
\text { 29. INDIAN HEAD } \\
\text { 30. KAMSACK } \\
\end{array}$ & $\begin{array}{l}2 \\
1\end{array}$ & $\begin{array}{l}1 \\
.4\end{array}$ & $\begin{array}{r}200 \\
82 \\
250\end{array}$ & $\begin{array}{r}5 \\
3 \\
15\end{array}$ & $\begin{array}{r}3 \\
2 \\
10 \\
18 \\
\end{array}$ & & $6-25$ & $6-25$ & & $\begin{array}{l}51.75 \\
51.75\end{array}$ & 51.75 & & $\begin{array}{c}6-25 \\
51-75 \\
26-50 \\
26-50 \\
\end{array}$ & $\begin{array}{r}6-25 \\
51-75 \\
\\
6-25 \\
26-50 \\
\end{array}$ & $\begin{array}{r}<5 \\
26-50 \\
6-25 \\
6-25 \\
\end{array}$ & \\
\hline $\begin{array}{l}\text { 31. KELVINGTON } \\
\text { 32. KENASTON } \\
\text { 33. KINDERSLEY } \\
\text { 34. KINLOCH } \\
\text { 35. KUTAWAGAN LAKE. } \\
\end{array}$ & $\begin{array}{l}2 \\
1 \\
\end{array}$ & $\begin{array}{l}1 \\
1 \\
\end{array}$ & $\begin{array}{r}60 \\
50 \\
85 \\
83 \\
200 \\
\end{array}$ & $\begin{array}{r}1 \\
2 \\
1.75 \\
3 \\
4 \\
\end{array}$ & $\begin{array}{r}1 \\
5 \\
.5 \\
10\end{array}$ & $<5$ & $26-50$ & & $26-50$ & $\begin{array}{r}<5 \\
6.25 \\
\\
51.75\end{array}$ & $6-25$ & & $\begin{array}{r}51.75 \\
6.25 \\
51.75 \\
\\
>75 \\
\end{array}$ & $\begin{array}{r}6-25 \\
26-50 \\
6-25 \\
<5 \\
\end{array}$ & $\begin{array}{l}<5 \\
<5\end{array}$ & \\
\hline $\begin{array}{l}\text { 36. LARONGE } \\
\text { 37. LASTMTN. LAKENWA } \\
\text { 38. LEADER } \\
\text { 39. LNELONG } \\
\text { 40. LOON LAKE }\end{array}$ & $\begin{array}{l}6 \\
3 \\
6\end{array}$ & $\begin{array}{r}1 \\
2 \\
1.5\end{array}$ & $\begin{array}{r}84 \\
163 \\
35 \\
10\end{array}$ & $\begin{array}{r}4.5 \\
4 \\
1.5 \\
2\end{array}$ & $\begin{array}{l}1 \\
5 \\
3 \\
\end{array}$ & $6-25$ & $51-75$ & $51-75$ & & $6-25$ & $\begin{array}{r}6-25 \\
3\end{array}$ & $\begin{array}{l}6-25 \\
6-25\end{array}$ & $\begin{array}{r}>75 \\
<5 \\
<5 \\
\end{array}$ & $\begin{array}{r}6-25 \\
26-50 \\
\end{array}$ & $>75$ & $<5$ \\
\hline
\end{tabular}


Table 2. COUNT COVERAGE (continued) ("indicates counts which conform to the North American standard of all day counts covering a 15-mile diameter circle)

\begin{tabular}{|c|c|c|c|c|c|c|c|c|c|c|c|c|c|c|c|c|}
\hline COUNT NAME & $\begin{array}{l}\overrightarrow{8} \\
\frac{4}{8} \\
E \\
x\end{array}$ & $\begin{array}{l}\overline{8} \\
4 \\
8 \\
5 \\
5 \\
\overline{8}\end{array}$ & $\begin{array}{l}\frac{\omega}{\frac{\omega}{2}} \\
\frac{.5}{5} \\
\frac{5}{5} \\
\frac{E}{x}\end{array}$ & $\begin{array}{l}\frac{0}{0} \\
\frac{0}{5} \\
\frac{0}{0} \\
0 \\
\frac{5}{3} \\
\frac{3}{1}\end{array}$ & 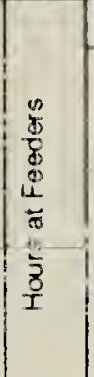 & $\begin{array}{l}\frac{8}{8} \\
\frac{8}{3} \\
\frac{0}{0} \\
\frac{3}{3} \\
\frac{3}{5}\end{array}$ & $\begin{array}{l}\frac{0}{8} \\
\frac{1}{3} \\
\frac{3}{8} \\
\frac{x}{2} \\
\frac{2}{2}\end{array}$ & $\begin{array}{l}\frac{1}{8} \\
8 \\
3 \\
3 \\
3 \\
3 \\
0 \\
0 \\
0 \\
0 \\
0\end{array}$ & 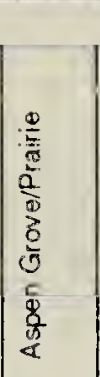 & 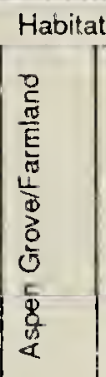 & 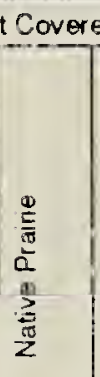 & 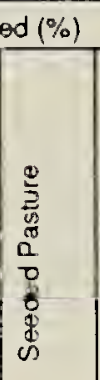 & 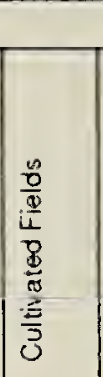 & है & $\frac{5}{5}$ & $\frac{\frac{5}{\pi}}{\sum^{\frac{5}{5}}}$ \\
\hline $\begin{array}{l}\text { 41. LOVE-TOPCH RIVER } \\
\text { 42. LUSELAND } \\
\text { 43. MACDOWALL } \\
\text { 44. MARSDEN } \\
\text { 45. MEADOW LAKE }\end{array}$ & $\begin{array}{r}1 \\
3 \\
4 \\
6.5 \\
4\end{array}$ & $\begin{array}{r}3 \\
1.5 \\
2 \\
3.5 \\
3.5 \\
\end{array}$ & $\begin{array}{r}30 \\
82 \\
45 \\
90 \\
110 \\
\end{array}$ & $\begin{array}{r}2 \\
2.5 \\
2\end{array}$ & $\begin{array}{r}18 \\
2 \\
3 \\
3 \\
1 \\
\end{array}$ & 6.25 & $\begin{array}{c}6-25 \\
6-25 \\
26-50\end{array}$ & $26-50$ & $6-25$ & $\begin{array}{r}51.75 \\
6-25\end{array}$ & & $<5$ & $\mid \begin{array}{r}6-25 \\
26-50 \\
6-25 \\
26-50 \\
6-25\end{array}$ & $\begin{array}{r}26-50 \\
6-25 \\
26-50\end{array}$ & $\begin{array}{r}26-50 \\
6-25 \\
26-50\end{array}$ & \\
\hline $\begin{array}{l}\text { 46. MELFORT } \\
\text { 47. MELVILLE } \\
\text { 48. MISSIN:PE } \\
\text { 49. MOOSE JAW } \\
\text { 50. MOOSE MOUNTAIN. }\end{array}$ & $\begin{array}{l}4 \\
3\end{array}$ & $\begin{array}{l}2 \\
3\end{array}$ & $\begin{array}{r}5 \\
166 \\
\end{array}$ & 6 & $\begin{array}{c}8 \\
10 \\
7 \\
2\end{array}$ & $<5$ & $<5$ & $26-50$ & $<5$ & $51-75$ & $<5$ & $<5$ & $\begin{array}{r}26-50 \\
6-25 \\
6-25\end{array}$ & $\begin{array}{r}26-50 \\
>75\end{array}$ & $\left|\begin{array}{r}26-50 \\
<5 \\
6-25 \\
6-25\end{array}\right|$ & 51.75 \\
\hline $\begin{array}{l}\text { 51. NiCQLLE FLATS } \\
\text { 52. NIPAWIN } \\
\text { 53. PIKELAKE } \\
\text { 54. PORCUPINE PLAIN } \\
\text { 55. PRINCE ALBERT (A) }\end{array}$ & \begin{tabular}{r|}
3 \\
5 \\
18.5 \\
1
\end{tabular} & $\begin{array}{l}.5 \\
7 \\
.5\end{array}$ & $\begin{array}{r}80 \\
10 \\
356 \\
60\end{array}$ & $\begin{array}{r}3 \\
1.5 \\
14.25 \\
1\end{array}$ & $\begin{array}{l}6 \\
3 \\
3 \\
2 \\
\end{array}$ & & \begin{tabular}{r|}
$6-25$ \\
$<5$ \\
$6-25$
\end{tabular} & 6-25 & $<5$ & 4 & $<5$ & $<5$ & $\begin{array}{r}26-50 \\
6-25\end{array}$ & $\begin{array}{r}<5 \\
26-50\end{array}$ & $\begin{array}{r}<5 \\
51-75 \\
26-50\end{array}$ & $<5$ \\
\hline $\begin{array}{l}\text { 56. PRINCE ALBERT (B) } \\
\text { 57. PRINCE ALBERT N.P. } \\
\text { 58. OUAPPELLE VAL. DAM' } \\
\text { 59. RAYMORE. } \\
\text { 60. REGINA. }\end{array}$ & $\begin{array}{r}4 \\
13.5 \\
2 \\
216 \\
29 \\
\end{array}$ & $\begin{array}{r}8 \\
2.1 \\
8 \\
19.5 \\
\end{array}$ & $\begin{array}{r}174.5 \\
45 \\
237 \\
5 \\
292\end{array}$ & $\begin{array}{r}6.75 \\
2.5 \\
6.3 \\
3 \\
30.5 \\
\end{array}$ & $\begin{array}{r}10 \\
5\end{array}$ & $\begin{array}{r}<5 \\
6-25\end{array}$ & $|26-50|$ & $\begin{array}{r}<5 \\
6-25 \\
26-50\end{array}$ & $6-25$ & $\mid$\begin{tabular}{c|}
$<5$ \\
$51-75$ \\
$51-75$
\end{tabular} & & $6-25$ & $\begin{array}{r}6-25 \\
26-50\end{array}$ & $\begin{array}{l}6-25 \\
6-25\end{array}$ & $\begin{array}{r}>75 \\
6-25 \\
6-25 \\
<5\end{array}$ & $\begin{array}{l}<5 \\
<5 \\
<5\end{array}$ \\
\hline $\begin{array}{l}\text { 61. REGINA BEACH } \\
\text { 62. ROUND LAKE } \\
\text { 63. SASKATOON } \\
\text { 64. SCOTT. } \\
\text { 65. SKULL CKEEK }\end{array}$ & $\begin{array}{r}75.4 \\
6 \\
16\end{array}$ & $\begin{array}{r}40 \\
3\end{array}$ & $\begin{array}{r}65 \\
35 \\
912.3 \\
155 \\
130 \\
\end{array}$ & $\begin{array}{r}1 \\
50.25 \\
5.5 \\
8 \\
\end{array}$ & $\begin{array}{r}2 \\
38\end{array}$ & & & $26-50$ & $26-50$ & $6-25$ & $6-25$ & $<5$ & $\begin{array}{l}26-50 \\
26-50 \\
26-50\end{array}$ & $\begin{array}{l}26-50 \\
26-50\end{array}$ & $\begin{array}{r}<5 \\
6-25\end{array}$ & $\begin{array}{r}<5 \\
6-25\end{array}$ \\
\hline $\begin{array}{l}\text { 66. SNOWDEN } \\
\text { 67. SOMME-NEELY LAKE } \\
\text { 63. SPNLDING } \\
\text { 69. SPINNEY HILL. } \\
\text { 70. SPRING VALLEY }\end{array}$ & $\begin{array}{l}2 \\
1 \\
8 \\
1 \\
3\end{array}$ & $\begin{array}{r}1 \\
.5 \\
3 \\
.45 \\
1.75 \\
\end{array}$ & $\begin{array}{r}102 \\
60 \\
50 \\
114 \\
104 \\
\end{array}$ & $\begin{array}{r}4 \\
1.5 \\
\end{array}$ & $\begin{array}{r}2.5 \\
2 \\
2\end{array}$ & $<5$ & $6-25$ & $6-25$ & & 51.75 & $6-25$ & $6-25$ & $\begin{array}{l}6-25 \\
6-25\end{array}$ & $\begin{array}{r}26-50 \\
>75 \\
6-25 \\
<5 \\
6-25\end{array}$ & $\begin{array}{r}6-25 \\
26-50 \\
<5\end{array}$ & \\
\hline $\begin{array}{l}\text { 71. SPRUCE HOME } \\
\text { 72. SOUAWRAPIDS } \\
\text { 73. SWIFI CURRENT } \\
\text { 74. TISDALE } \\
\text { 75. TURTLE LAKE }\end{array}$ & $\begin{array}{r}15 \\
4\end{array}$ & $\begin{array}{r}8 \\
1.5 \\
20\end{array}$ & $\begin{array}{r}10 \\
90 \\
189 \\
1 \\
30 \\
\end{array}$ & $\begin{array}{r}14 \\
9.5\end{array}$ & 29.5 & 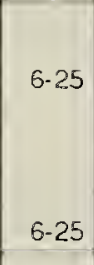 & $\begin{array}{l}51-75 \\
26-50\end{array}$ & $6-25$ & & $6-25$ & $6-25$ & & $\begin{array}{r}26-50 \\
<5 \\
51-75\end{array}$ & $\begin{array}{r}<5 \\
6-25\end{array}$ & $<5$ & $\begin{array}{l}<5 \\
<5\end{array}$ \\
\hline $\begin{array}{l}\text { 76. VAL MARIE } \\
\text { 77. WEYBURN' } \\
\text { 78. WHITE BEAR } \\
\text { 79. WHITEWOOD } \\
\text { 80. YORIKTON }\end{array}$ & $\begin{array}{r}3 \\
10.4 \\
8 \\
.5 \\
20\end{array}$ & $\begin{array}{r}1 \\
2.5 \\
2.5 \\
.75 \\
5\end{array}$ & $\begin{array}{c}92.7 \\
287\end{array}$ & $\begin{array}{r}4 \\
14.25\end{array}$ & $\begin{array}{r}1.5 \\
.5 \\
.75\end{array}$ & $6-25$ & $6-25$ & $6 \cdot 25$ & 6.25 & 51.75 & $\begin{array}{r}51.75 \\
6-25 \\
3\end{array}$ & $<5$ & $\begin{array}{r}6-25 \\
26-50 \\
26-50 \\
6-25\end{array}$ & $\begin{array}{r}<5 \\
6-25 \\
6-25 \\
<5\end{array}$ & $\mid \begin{array}{r}<5 \\
6-25 \\
\\
6-25 \\
51-75 \\
\end{array}$ & $<5$ \\
\hline
\end{tabular}


Table 3-1. SPECIES RECORDED FROM FIVE OR MORE LOCALITIES ( + = a species seen during the count period but not on count day)

\begin{tabular}{|c|c|c|c|c|c|c|c|c|c|c|c|}
\hline & & & & & LOCAT & ION ANI & D DATE & & & & \\
\hline SPECIES & 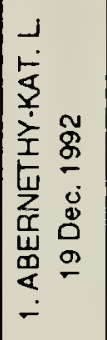 & 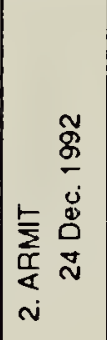 & 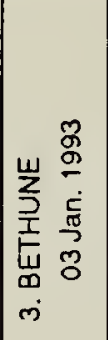 & 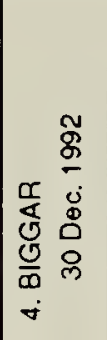 & 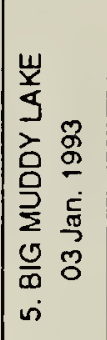 & 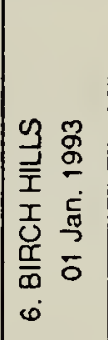 & 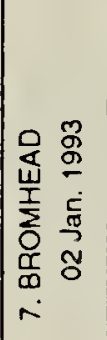 & 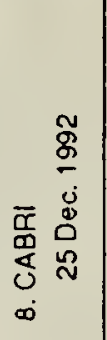 & 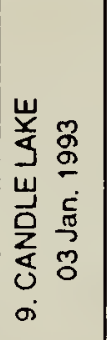 & 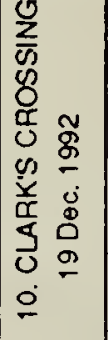 & 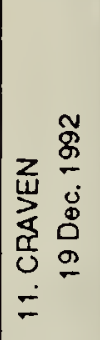 \\
\hline $\begin{array}{l}\text { CANADA GOOSE } \\
\text { MALLARD } \\
\text { COMMON GOLDENEYE } \\
\text { BALD EAGLE } \\
\text { NORTHERN GOSHAWK }\end{array}$ & & 2 & & & & & & & 1 & $\begin{array}{l}1 \\
1\end{array}$ & \\
\hline $\begin{array}{l}\text { GOLDEN EAGLE } \\
\text { MERLIN } \\
\text { GYRFALCON } \\
\text { PRAIRIE FALCON } \\
\text { GRAY PARTRIDGE }\end{array}$ & + & & 16 & 51 & $\begin{array}{r}2 \\
17\end{array}$ & & 1 & 22 & & $\begin{array}{c}1 \\
50\end{array}$ & 1 \\
\hline $\begin{array}{l}\text { RING-NECKED PHEASANT } \\
\text { RUFFED GROUSE } \\
\text { SHARP-TAILED GROUSE } \\
\text { ROCK DOVE } \\
\text { GREAT HORNED OWL }\end{array}$ & $\begin{array}{r}+ \\
42 \\
2\end{array}$ & $\begin{array}{r}4 \\
17\end{array}$ & 6 & $\begin{array}{r}40 \\
218 \\
2\end{array}$ & $\begin{array}{l}4 \\
9 \\
5\end{array}$ & 100 & $\begin{array}{r}13 \\
16 \\
5 \\
4\end{array}$ & $\begin{array}{r}3 \\
12 \\
83 \\
1\end{array}$ & 1 & $\begin{array}{r}18 \\
186 \\
1\end{array}$ & $\begin{array}{r}1 \\
2 \\
16\end{array}$ \\
\hline $\begin{array}{l}\text { SNOWY OWL. } \\
\text { DOWNY WOODPECKER } \\
\text { HAIRY WOODPECKER } \\
\text { NORTHERN FLICKER } \\
\text { PILEATED WOODPECKER }\end{array}$ & $\begin{array}{l}+ \\
+ \\
1\end{array}$ & $\begin{array}{l}2 \\
1\end{array}$ & 1 & $\begin{array}{l}3 \\
2\end{array}$ & 1 & $\begin{array}{l}1 \\
1\end{array}$ & & 2 & $\begin{array}{l}6 \\
4\end{array}$ & 2 & $\begin{array}{l}4 \\
4\end{array}$ \\
\hline $\begin{array}{l}\text { HORNED LARK } \\
\text { GRAY JAY } \\
\text { BLUE JAY } \\
\text { BLACK.BILLED MAGPIE } \\
\text { COMMON RAVEN }\end{array}$ & $\begin{array}{r}4 \\
+\end{array}$ & $\begin{array}{r}7 \\
4 \\
14 \\
21\end{array}$ & + & $\begin{array}{r}24 \\
\\
3 \\
125 \\
4\end{array}$ & 70 & $\begin{array}{r}2 \\
2 \\
21 \\
9\end{array}$ & 5 & 8 & $\begin{array}{r}16 \\
5 \\
11 \\
92\end{array}$ & 201 & $\begin{array}{r}9 \\
1 \\
75\end{array}$ \\
\hline $\begin{array}{l}\text { BLACK-CAPPED CHICKADEE } \\
\text { BOREAL CHICKADEE } \\
\text { RED-BREASTED NUTHATCH } \\
\text { WHITE-BREASTED NUTHATCH } \\
\text { GOLDEN-CRO WNED KINGLET }\end{array}$ & 4 & $\begin{array}{r}18 \\
1\end{array}$ & + & 25 & 4 & 23 & & & $\begin{array}{r}63 \\
23 \\
5 \\
3\end{array}$ & 31 & 43 \\
\hline $\begin{array}{l}\text { AMERICAN ROBIN } \\
\text { BOHEMIAN WAXWING } \\
\text { CEDAR WAXWING } \\
\text { NORTHERN SHRIKE } \\
\text { EUROPEAN STARLING }\end{array}$ & + & & & 9 & $\begin{array}{r}10 \\
1 \\
13 \\
\end{array}$ & 80 & & + & 1 & $\begin{array}{r}49 \\
6 \\
12 \\
\end{array}$ & $\begin{array}{r}2 \\
15\end{array}$ \\
\hline $\begin{array}{l}\text { DARK-EYED JUNCO } \\
\text { LAPLANDLONGSPUR } \\
\text { SNOWBUNTING } \\
\text { RUSTY BLACKBIRD } \\
\text { PINE GROSBEAK } \\
\end{array}$ & 40 & $\begin{array}{r}342 \\
4 \\
\end{array}$ & 20 & 309 & $\begin{array}{r}5 \\
618 \\
2\end{array}$ & 95 & $\begin{array}{l}263 \\
335\end{array}$ & 230 & 7 & 886 & 207 \\
\hline $\begin{array}{l}\text { COMMON REDPOLL } \\
\text { HOARY REDPOLL } \\
\text { PINE SISKIN } \\
\text { EVENING GROSBEAK } \\
\text { HOUSE SPARROW }\end{array}$ & 40 & 160 & 30 & $\begin{array}{r}18 \\
5\end{array}$ & 222 & $\begin{array}{l}33 \\
49\end{array}$ & 14 & 60 & 37 & 1276 & 123 \\
\hline NO. INDIV. TABLES $3 \& 4$ & 0 & 6 & 0 & 1 & 10 & 0 & 0 & 1 & 5 & 0 & 0 \\
\hline NO. SPECIES COUNT DAY & 8 & 19 & 6 & 19 & 21 & 14 & 11 & 10 & 18 & 18 & 16 \\
\hline NO. SPECIES COUNT PERIOD & 14 & 19 & 9 & 21 & 21 & 14 & 11 & 11 & 18 & 18 & 17 \\
\hline NO. SPECIES TABLES $3 \& 4$ & 0 & 4 & 0 & 1 & 3 & 0 & 0 & 1 & 2 & 0 & 0 \\
\hline NO. INDIV. COUNT DAY & 134 & 604 & 81 & 1754 & 1459 & 419 & 2620 & 428 & 281 & 2779 & 515 \\
\hline
\end{tabular}




\section{Table 3-2. SPECIES RECORDED FROM FIVE OR MORE LOCALITIES}

( $+=\mathrm{a}$ species seen during the count period but not on count day)

\begin{tabular}{|c|c|c|c|c|c|c|c|c|c|c|c|}
\hline & & & & & LOCAT & ION ANC & DATE & & & & \\
\hline SPECIES & 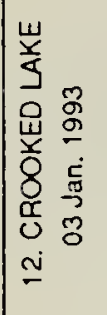 & 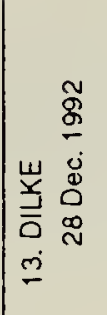 & 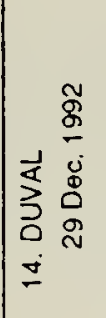 & 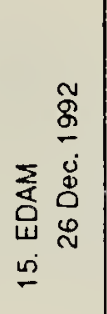 & 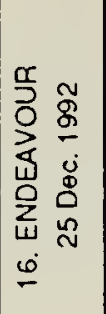 & 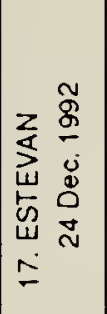 & 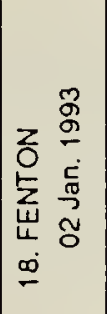 & 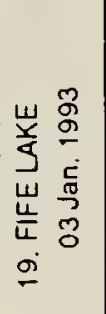 & 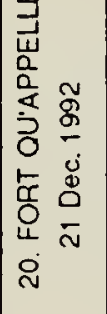 & 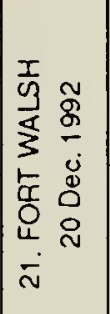 & 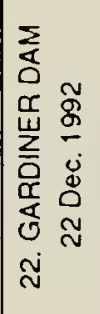 \\
\hline $\begin{array}{l}\text { CANADA GOOSE } \\
\text { MALLARD } \\
\text { COMMON GOLDENEYE } \\
\text { BALD EAGLE } \\
\text { NORTHERN GOSHAYK } \\
\end{array}$ & 1 & + & & + & + & $\begin{array}{r}188 \\
1988 \\
58\end{array}$ & & $\begin{array}{r}2500 \\
3\end{array}$ & $\begin{array}{r}1 \\
72 \\
13 \\
1\end{array}$ & $\begin{array}{l}5 \\
3 \\
1 \\
\end{array}$ & $\begin{array}{r}1 \\
3170 \\
20 \\
9\end{array}$ \\
\hline $\begin{array}{l}\text { GOLDEN EAGLE } \\
\text { MERLIN } \\
\text { GYRFALCON } \\
\text { PRAIRIE FALCON } \\
\text { GRAY PARTRIDGE } \\
\end{array}$ & & 68 & 1 & & & + & & $\begin{array}{r}1 \\
1 \\
1 \\
1 \\
15 \\
\end{array}$ & + & 17 & $\begin{array}{r}4 \\
+ \\
25 \\
\end{array}$ \\
\hline $\begin{array}{l}\text { RING-NECKED PHEASANT } \\
\text { RUFFED GROUSE } \\
\text { SHARP-TAILED GROUSE } \\
\text { ROCK DOVE } \\
\text { GREAT HORNED OWL } \\
\end{array}$ & 1 & $\begin{array}{l}+ \\
8\end{array}$ & $\begin{array}{r}4 \\
11\end{array}$ & V & 1 & $\begin{array}{r}1 \\
76 \\
1 \\
\end{array}$ & 30 & 1 & $\begin{array}{l}+ \\
+ \\
+ \\
6 \\
+\end{array}$ & $\begin{array}{l}3 \\
5 \\
2 \\
\end{array}$ & $\begin{array}{r}29 \\
35 \\
4 \\
\end{array}$ \\
\hline $\begin{array}{l}\text { SNOWY OWL } \\
\text { DOWNY WOODPECKER } \\
\text { HAIRY WOODPECKER } \\
\text { NORTHERN FLICKER } \\
\text { PII.EATED WOODPECKER }\end{array}$ & $\begin{array}{l}7 \\
4\end{array}$ & + & $\begin{array}{l}1 \\
1\end{array}$ & $\begin{array}{l}1 \\
2 \\
2\end{array}$ & 1 & 1 & $\begin{array}{l}1 \\
3\end{array}$ & 1 & $\begin{array}{l}+ \\
4 \\
8\end{array}$ & $\begin{array}{l}1 \\
5 \\
6\end{array}$ & $\begin{array}{l}3 \\
1 \\
2\end{array}$ \\
\hline $\begin{array}{l}\text { HORNED LARK } \\
\text { GRAY JAY } \\
\text { BLUE JAY } \\
\text { BLACK.BILLED MAGPIE } \\
\text { COMMON RAVEN } \\
\end{array}$ & 8 & 20 & $\begin{array}{r}25 \\
\\
2 \\
16\end{array}$ & $\begin{array}{r}4 \\
+ \\
\end{array}$ & $\begin{array}{r}5 \\
24 \\
\end{array}$ & 95 & $\begin{array}{r}4 \\
63 \\
11 \\
\end{array}$ & $\begin{array}{c}300 \\
11\end{array}$ & $\begin{array}{r}10 \\
14 \\
+ \\
\end{array}$ & $\begin{array}{r}1 \\
131\end{array}$ & $\begin{array}{r}26 \\
1 \\
87\end{array}$ \\
\hline $\begin{array}{l}\text { BLACK-CAPPED CHICKADEE } \\
\text { BOREAL CHICKADEE } \\
\text { RED-BREASTED NUTHATCH } \\
\text { WHITE-BREASTED NUTHATCH } \\
\text { GOLDEN-CROWNED KINGLET }\end{array}$ & 8 & + & 9 & 8 & 3 & 8 & 12 & & 8 & $\begin{array}{r}109 \\
18 \\
9 \\
\end{array}$ & 13 \\
\hline $\begin{array}{l}\text { AMERICAN ROBIN } \\
\text { BOHEMIAN WAXWING } \\
\text { CEDAR WAXWING } \\
\text { NORTHERN SHRIKE } \\
\text { EUROPEAN STARLING } \\
\end{array}$ & 1 & & 11 & & & 1 & & + & $\begin{array}{l}3 \\
6 \\
1\end{array}$ & 3 & $\begin{array}{r}61 \\
1 \\
8 \\
\end{array}$ \\
\hline $\begin{array}{l}\text { DARK-EYED JUNCO } \\
\text { LAPLAND LONGSPUR } \\
\text { SNOWBUNTING } \\
\text { RUSTY BLACKBIRD } \\
\text { PINE GROSBEAK } \\
\end{array}$ & 150 & + & 374 & 1 & 150 & $\begin{array}{r}1 \\
275\end{array}$ & 174 & & $\begin{array}{c}1 \\
10\end{array}$ & $\begin{array}{r}3 \\
344 \\
11\end{array}$ & $\begin{array}{r}194 \\
8 \\
\end{array}$ \\
\hline $\begin{array}{l}\text { COMMON REDPOLL } \\
\text { HOARY REDPOLL } \\
\text { PINE SISKIN } \\
\text { EVENING GROSBEAK } \\
\text { HOUSE SPARROW }\end{array}$ & 21 & 62 & 232 & 10 & $\begin{array}{l}5 \\
2 \\
\end{array}$ & 75 & 18 & 60 & $\begin{array}{r}+ \\
+ \\
124 \\
\end{array}$ & 135 & 536 \\
\hline NO. INDN. TABLES $3 \& 4$ & 0 & 0 & 0 & 0 & 1 & 28 & 0 & 0 & 3 & 86 & 258 \\
\hline NO. SPECIES COUNT DAY & 11 & 6 & 14 & 7 & 9 & 21 & 9 & 13 & 20 & 28 & 25 \\
\hline NO. SPECIES COUNT PERIOD & 13 & 12 & 15 & 11 & 10 & 22 & 10 & 15 & 32 & 28 & 26 \\
\hline NO. SPECIES TABLES $3 \& 4$ & 2 & 0 & 0 & 0 & 1 & 4 & 0 & 0 & 5 & 5 & 3 \\
\hline NO. INDIV. COUNT DAY & 268 & 176 & 690 & 28 & 192 & 2808 & 316 & 2899 & 357 & 937 & 4496 \\
\hline
\end{tabular}


Table 3-3. SPECIES RECORDED FROM FIVE OR MORE LOCALITIES ( $+=$ a species seen during the count period but not on count day)

\begin{tabular}{|c|c|c|c|c|c|c|c|c|c|c|c|}
\hline & & & & & LOCATI & ION AND & D DATE & & & & \\
\hline SPECIES & 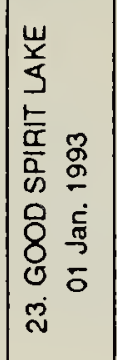 & 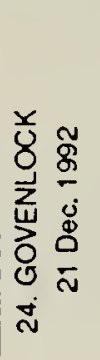 & 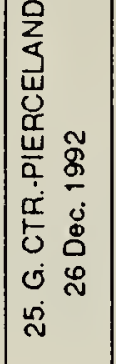 & 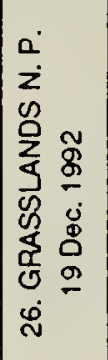 & 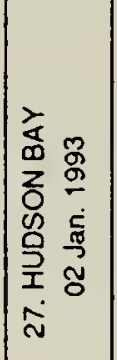 & 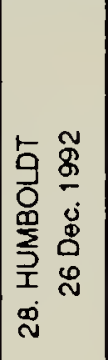 & 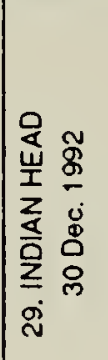 & 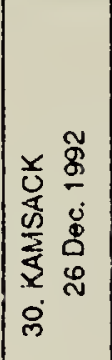 & 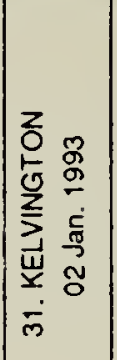 & 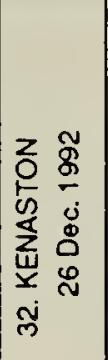 & 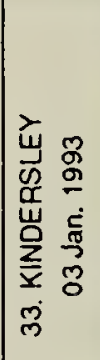 \\
\hline $\begin{array}{l}\text { CANADA GOOSE } \\
\text { MALLARD } \\
\text { COMMON GOLDENEYE } \\
\text { BALD EAGLE } \\
\text { NORTHERN GOSHAWK }\end{array}$ & & & & 1 & & & & & & & \\
\hline $\begin{array}{l}\text { GOLDEN EAGLE } \\
\text { MERLIN } \\
\text { GYRFALCON } \\
\text { PRAIRIE FALCON } \\
\text { GRAY PARTRIDGE }\end{array}$ & & $\begin{array}{r}5 \\
27\end{array}$ & & 6 & & & 10 & 10 & + & 18 & \\
\hline $\begin{array}{l}\text { RING-NECKED PHEASANT } \\
\text { RUFFED GROUSE } \\
\text { SHARP-TAILED GROUSE } \\
\text { ROCK DOVE } \\
\text { GREAT HORNED OWL }\end{array}$ & $\begin{array}{l}+ \\
2\end{array}$ & $\begin{array}{l}4 \\
5\end{array}$ & & 9 & + & 112 & $\begin{array}{l}8 \\
2 \\
2\end{array}$ & 18 & & $\begin{array}{r}30 \\
+\end{array}$ & \\
\hline $\begin{array}{l}\text { SNOWY OWL } \\
\text { DOWN WOODPECKER } \\
\text { HAIRY WOODPECKER } \\
\text { NORTHERN FLICKER } \\
\text { PILEATED WOODPECKER }\end{array}$ & $\begin{array}{l}8 \\
9\end{array}$ & $\begin{array}{l}7 \\
1\end{array}$ & $\begin{array}{l}3 \\
7\end{array}$ & & $\begin{array}{l}2 \\
3\end{array}$ & 2 & $\begin{array}{l}1 \\
3 \\
2\end{array}$ & $\begin{array}{r}6 \\
12 \\
13\end{array}$ & $\begin{array}{l}4 \\
5\end{array}$ & 1 & 5 \\
\hline $\begin{array}{l}\text { HORNED LARK } \\
\text { GRAY JAY } \\
\text { BLUE JAY } \\
\text { BLACK.BILLED MMGPIE } \\
\text { COMMON RAVEN } \\
\end{array}$ & $\begin{array}{r}3 \\
24 \\
6 \\
\end{array}$ & 17 & \begin{tabular}{r|}
2 \\
12 \\
17 \\
101 \\
\end{tabular} & 29 & \begin{tabular}{r|r}
2 \\
18 \\
6 \\
23 \\
\end{tabular} & $\begin{array}{r}1 \\
15\end{array}$ & $\begin{array}{r}8 \\
39\end{array}$ & $\begin{array}{l}17 \\
38 \\
16 \\
38 \\
\end{array}$ & $\begin{array}{l}4 \\
8 \\
2 \\
\end{array}$ & & 2 \\
\hline $\begin{array}{l}\text { BLACK. CAPPED CHICKADEE } \\
\text { BOREAL CHICKADEE } \\
\text { RED.BREASTED NU THATCH } \\
\text { WHITE.BREASTED NUTHATCH } \\
\text { GOLDEN.CRO WNED KINGLET }\end{array}$ & 43 & 2 & 41 & & 42 & 32 & $\begin{array}{l}3 \\
5\end{array}$ & \begin{tabular}{r|}
140 \\
4 \\
15
\end{tabular} & 1 & 3 & \\
\hline $\begin{array}{l}\text { AMERICAN ROBIN } \\
\text { BOHEMIAN WAXWING } \\
\text { CEDAR WAXWING } \\
\text { NORTHERN SHRIKE } \\
\text { EUROPEAN STARLING }\end{array}$ & $\begin{array}{l}+ \\
7 \\
\end{array}$ & $\begin{array}{l}4 \\
6 \\
\end{array}$ & 3 & $\begin{array}{l}2 \\
2 \\
\end{array}$ & & 450 & 1 & 90 & & & 39 \\
\hline $\begin{array}{l}\text { DARK-EYED JUNCO } \\
\text { LAPLAND LONGSPUR } \\
\text { SNOWBUNTING } \\
\text { RUSTY BLACKBIRD } \\
\text { PINE GROSBEAK } \\
\end{array}$ & 38 & $\begin{array}{r}13 \\
1\end{array}$ & $\begin{array}{l}80 \\
21 \\
\end{array}$ & 1 & $\begin{array}{c}249 \\
61 \\
\end{array}$ & 45 & 330 & 70 & $\begin{array}{r}500 \\
3 \\
\end{array}$ & & \\
\hline $\begin{array}{l}\text { COMMON REDPOLL } \\
\text { HOARY REDPOLL } \\
\text { PINE SISKIN } \\
\text { EVENING GROSBEAK } \\
\text { HOUSE SPARROW } \\
\end{array}$ & \begin{tabular}{r|}
20 \\
2 \\
89 \\
\end{tabular} & 240 & $\begin{array}{r}1 \\
15 \\
\end{array}$ & 3 & $\begin{array}{r}21 \\
3 \\
106 \\
5 \\
\end{array}$ & 50 & 160 & $\begin{array}{r}10 \\
564\end{array}$ & $\begin{array}{r}28 \\
5 \\
\end{array}$ & 26 & 40 \\
\hline $\begin{array}{l}\text { NO. INDIV. TABLES } 3 \& 4 \\
\text { NO. SPECIES COUNT DAY } \\
\text { NO. SPECIES COUNT PERIOD } \\
\text { NO. SPECIES TABLES } 384 \\
\text { NO. INDIV. COUNT DAY }\end{array}$ & \begin{tabular}{r|}
0 \\
15 \\
18 \\
0 \\
271
\end{tabular} & $\begin{array}{r}9 \\
21 \\
21 \\
4 \\
1294\end{array}$ & \begin{tabular}{r|} 
\\
14 \\
15 \\
0 \\
393
\end{tabular} & $\begin{array}{r}19 \\
16 \\
16 \\
3 \\
80\end{array}$ & \begin{tabular}{r|}
0 \\
14 \\
17 \\
1 \\
546
\end{tabular} & \begin{tabular}{r|}
0 \\
10 \\
13 \\
0 \\
710
\end{tabular} & $\begin{array}{r}0 \\
16 \\
16 \\
0 \\
629\end{array}$ & $\begin{array}{r}1 \\
21 \\
21 \\
1 \\
1097\end{array}$ & $\begin{array}{r}0 \\
11 \\
12 \\
0 \\
580\end{array}$ & \begin{tabular}{r|}
0 \\
6 \\
8 \\
0 \\
79 \\
\end{tabular} & $\begin{array}{r}0 \\
4 \\
4 \\
0 \\
86 \\
\end{array}$ \\
\hline
\end{tabular}


Table 3-4. SPECIES RECORDED FROM FIVE OR MORE LOCALITIES $(t=a$ species seen during the count period but not on count day)

\begin{tabular}{|c|c|c|c|c|c|c|c|c|c|c|c|}
\hline & & & & & LOCAT & ION ANE & DDATE & & & & \\
\hline SPECIES & 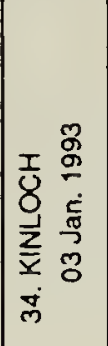 & 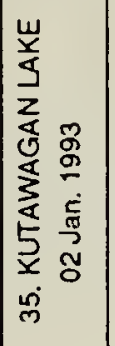 & 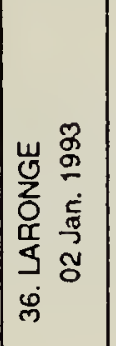 & 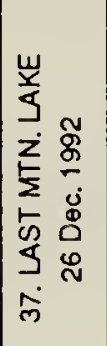 & 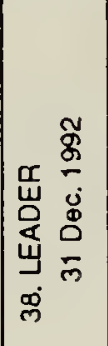 & 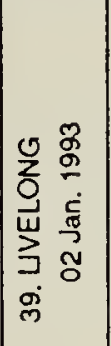 & 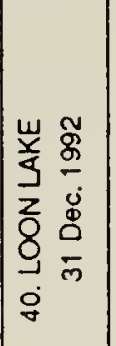 & 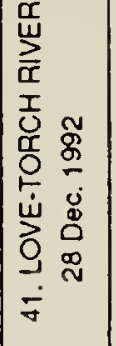 & 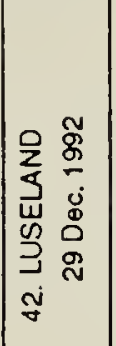 & 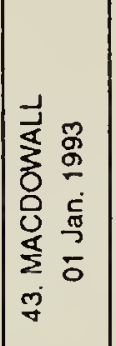 & 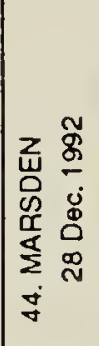 \\
\hline $\begin{array}{l}\text { CANADA GOOSE } \\
\text { MALLARD } \\
\text { COMMON GOLDENEYE } \\
\text { BALD EAGLE } \\
\text { NORTHERN GOSHAWK }\end{array}$ & & & & & 1 & & & 1 & & & \\
\hline $\begin{array}{l}\text { GOLDEN EAGLE } \\
\text { MERLIN } \\
\text { GYRFALCON } \\
\text { PRAIRIE FALCON } \\
\text { GRAY PARTRIDGE }\end{array}$ & & & & 6 & 1 & & & & 22 & & 33 \\
\hline $\begin{array}{l}\text { RING-NECKED PHEASANT } \\
\text { RUFFED GROUSE } \\
\text { SHARP-TAILED GROUSE } \\
\text { ROCK DOVE } \\
\text { GREAT HORNED OWL }\end{array}$ & 5 & $\begin{array}{l}6 \\
1\end{array}$ & & $\begin{array}{r}35 \\
127 \\
4\end{array}$ & $\begin{array}{r}21 \\
20 \\
1\end{array}$ & 50 & 2 & $\begin{array}{r}10 \\
1\end{array}$ & $\begin{array}{r}+ \\
34 \\
+\end{array}$ & & $\begin{array}{r}9 \\
25 \\
5\end{array}$ \\
\hline $\begin{array}{l}\text { SNOWY OWL } \\
\text { DOWNY WOODPECKER } \\
\text { HAIRY WOODPECKER } \\
\text { NORTHERN FLICKER } \\
\text { PILEATED WOODPECKER }\end{array}$ & 3 & 2 & $\begin{array}{l}+ \\
5\end{array}$ & 1 & 1 & $\begin{array}{l}5 \\
7\end{array}$ & 3 & $\begin{array}{l}2 \\
5\end{array}$ & $\begin{array}{l}1 \\
2 \\
1\end{array}$ & $\begin{array}{l}2 \\
2\end{array}$ & $\begin{array}{r}1 \\
11 \\
9\end{array}$ \\
\hline $\begin{array}{l}\text { HORNED LARK } \\
\text { GRAY JAY } \\
\text { BLUE JAY } \\
\text { BLACK.BILLED MMGPE } \\
\text { COMMON RAVEN } \\
\end{array}$ & $\begin{array}{l}2 \\
2 \\
1 \\
4 \\
\end{array}$ & $\begin{array}{r}1 \\
39\end{array}$ & $\begin{array}{r}5 \\
1 \\
2 \\
123 \\
\end{array}$ & 61 & 28 & $\begin{array}{r}18 \\
5 \\
4 \\
\end{array}$ & $\begin{array}{l}2 \\
3 \\
3 \\
\end{array}$ & $\begin{array}{r}4 \\
13 \\
+ \\
17 \\
\end{array}$ & $\begin{array}{r}3 \\
13\end{array}$ & $\begin{array}{r}6 \\
50 \\
25 \\
\end{array}$ & $\begin{array}{r}8 \\
17\end{array}$ \\
\hline $\begin{array}{l}\text { BLACK.CAPPED CHICKADEE } \\
\text { BOREAL CHICKADEE } \\
\text { RED.BREASTED NUTHATCH } \\
\text { WHITE-BREASTED NUTHATCH } \\
\text { GOLDEN.CRO WNED KINGLET }\end{array}$ & 16 & 3 & $\begin{array}{r}17 \\
3\end{array}$ & & 1 & 28 & 9 & $\begin{array}{r}51 \\
2\end{array}$ & 6 & 20 & 107 \\
\hline $\begin{array}{l}\text { AMERICAN ROBIN } \\
\text { BOHEMIAN WAXWING } \\
\text { CEDAR WAXWING } \\
\text { NORTHERN SHRIKE } \\
\text { EUROPEAN STARLING } \\
\end{array}$ & & & & 5 & 60 & 6 & & & 19 & 20 & 20 \\
\hline $\begin{array}{l}\text { DARK-EYED JUNCO } \\
\text { LAPLAND LONGSPUR } \\
\text { SNOW BUNTING } \\
\text { RUSTY BLACKBIRD } \\
\text { PINE GROSBEAK } \\
\end{array}$ & 35 & 130 & 15 & $\begin{array}{r}226 \\
1\end{array}$ & & 50 & 1 & 27 & + & 1 & 120 \\
\hline $\begin{array}{l}\text { COMMON REDPOLL } \\
\text { HOARY REDPOLL } \\
\text { PINE SISKIN } \\
\text { EVENING GROSBEAK } \\
\text { HOUSE SPARROW } \\
\end{array}$ & $\begin{array}{l}10 \\
14 \\
\end{array}$ & 370 & 14 & 385 & 6 & $\begin{array}{l}20 \\
40 \\
\end{array}$ & & $\begin{array}{r}277 \\
3 \\
\end{array}$ & 171 & $\begin{array}{r}25 \\
1 \\
\end{array}$ & 386 \\
\hline $\begin{array}{l}\text { NO. INDIV. TABLES } 384 \\
\text { NO. SPECIES COUNT DAY } \\
\text { NO. SPECIES COUNT PERIOD } \\
\text { NO. SPECIES TABLES } 384 \\
\text { NO. INDIV. COUNT DAY }\end{array}$ & $\begin{array}{r}0 \\
11 \\
13 \\
0 \\
115\end{array}$ & \begin{tabular}{r|}
0 \\
8 \\
8 \\
0 \\
552
\end{tabular} & $\begin{array}{r}0 \\
10 \\
11 \\
0 \\
189\end{array}$ & $\begin{array}{r}0 \\
11 \\
11 \\
0 \\
852\end{array}$ & $\begin{array}{r}1 \\
11 \\
11 \\
1 \\
141\end{array}$ & \begin{tabular}{r|} 
\\
12 \\
12 \\
0 \\
273
\end{tabular} & $\begin{array}{r}0 \\
7 \\
7 \\
0 \\
23\end{array}$ & \begin{tabular}{r|}
0 \\
14 \\
17 \\
0 \\
417
\end{tabular} & $\begin{array}{r}0 \\
10 \\
13 \\
0 \\
272\end{array}$ & $\begin{array}{r}1 \\
13 \\
14 \\
1 \\
158\end{array}$ & $\begin{array}{r}5 \\
17 \\
17 \\
1 \\
770\end{array}$ \\
\hline
\end{tabular}


Table 3-5. SPECIES RECORDED FROM FIVE OR MORE LOCALITIES $(t=$ a species seen during the count period but not on count day)

\begin{tabular}{|c|c|c|c|c|c|c|c|c|c|c|c|}
\hline & & & & & LOCAT & IION AN & D DATE & & & & \\
\hline SPECIES & 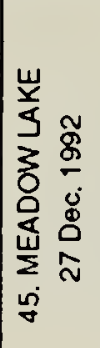 & 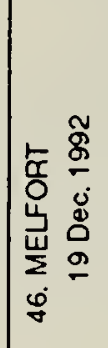 & 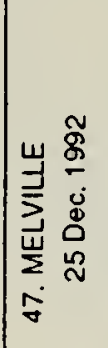 & 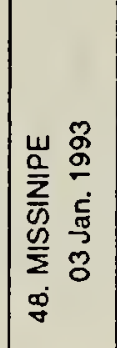 & 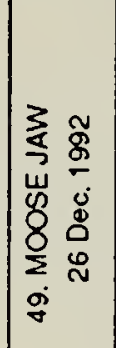 & 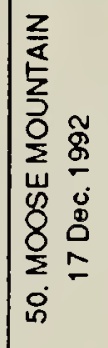 & 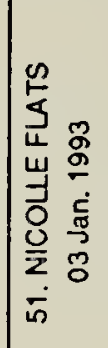 & 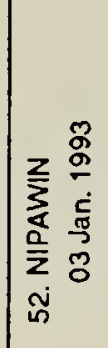 & 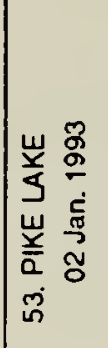 & 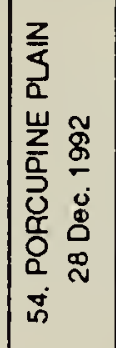 & 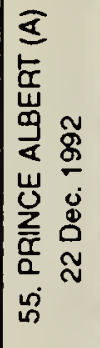 \\
\hline $\begin{array}{l}\text { CANADA GOOSE } \\
\text { MALLARD } \\
\text { COMMON GOLDENEYE } \\
\text { BALD EAGLE } \\
\text { NORTHERN GOSHAWK }\end{array}$ & & & + & & & 1 & & & 1 & & \\
\hline $\begin{array}{l}\text { GOLDEN EAGLE } \\
\text { MERLIN } \\
\text { GYRFALCON } \\
\text { PRAIRIE FALCON } \\
\text { GRAY PARTRIOGE }\end{array}$ & & & & & 1 & & 27 & & 1 & & \\
\hline $\begin{array}{l}\text { RING-NECKED PHEASANT } \\
\text { RUFFED GROUSE } \\
\text { SHARP-TALED GROUSE } \\
\text { ROCK DOVE } \\
\text { GREAT HORNED OWL }\end{array}$ & & & 20 & & $\begin{array}{r}60 \\
1\end{array}$ & $\begin{array}{l}5 \\
2 \\
1\end{array}$ & & & $\begin{array}{r}4 \\
128 \\
3 \\
2\end{array}$ & + & \\
\hline $\begin{array}{l}\text { SNOWY OWL } \\
\text { DOWNY WOODPECKER } \\
\text { HAIRY WOODPECKER } \\
\text { NORTHERN FLICKER } \\
\text { PILEATED WOODPECKER }\end{array}$ & $\begin{array}{l}3 \\
3\end{array}$ & & $\begin{array}{l}1 \\
1\end{array}$ & 1 & $\begin{array}{l}3 \\
2 \\
2\end{array}$ & $\begin{array}{r}9 \\
10\end{array}$ & 1 & $\begin{array}{l}2 \\
1\end{array}$ & $\begin{array}{l}10 \\
14\end{array}$ & + & $\begin{array}{l}2 \\
2\end{array}$ \\
\hline $\begin{array}{l}\text { HORNED LARK } \\
\text { GRAY JAY } \\
\text { BLUE JAY } \\
\text { BLACK.BILLED MAGPIE } \\
\text { COMMON RAVEN } \\
\end{array}$ & $\begin{array}{r}5 \\
3 \\
5 \\
28 \\
\end{array}$ & $\begin{array}{l}5 \\
8 \\
5 \\
\end{array}$ & $\begin{array}{l}1 \\
1 \\
1 \\
\end{array}$ & $\begin{array}{l}6 \\
4 \\
4 \\
\end{array}$ & 10 & $\begin{array}{r}14 \\
32 \\
. \quad 1 \\
\end{array}$ & 66 & $\begin{array}{r}2 \\
6 \\
1 \\
41 \\
\end{array}$ & $\begin{array}{r}20 \\
111 \\
\quad 3 \\
\end{array}$ & $\begin{array}{r}3 \\
9 \\
5 \\
29 \\
\end{array}$ & $\begin{array}{l}1 \\
4 \\
2 \\
\end{array}$ \\
\hline $\begin{array}{l}\text { BLACK-CAPPED CHICKADEE } \\
\text { BOREAL CHICKADEE } \\
\text { RED-BREASTED NUTHATCH } \\
\text { WHITE-BREASTED NUTHATCH } \\
\text { GOLDEN-CRO WNED KINGLET }\end{array}$ & $\begin{array}{r}20 \\
1\end{array}$ & 3 & 3 & $\begin{array}{l}6 \\
8\end{array}$ & $\begin{array}{l}1 \\
9\end{array}$ & $\begin{array}{r}72 \\
10 \\
8 \\
\end{array}$ & & $\begin{array}{l}2 \\
2\end{array}$ & \begin{tabular}{r|}
117 \\
2 \\
5
\end{tabular} & 2 & 10 \\
\hline $\begin{array}{l}\text { AMERICAN ROBIN } \\
\text { BOHEMIAN WAXWING } \\
\text { CEDAR WAXWING } \\
\text { NORTHERN SHRIKE } \\
\text { EUROPEAN STARLING } \\
\end{array}$ & 10 & 20 & + & & $\begin{array}{l}2 \\
5\end{array}$ & $\begin{array}{r}15 \\
1\end{array}$ & & 5 & $\begin{array}{r}159 \\
1\end{array}$ & & \\
\hline $\begin{array}{l}\text { DARK-EYED JUNCO } \\
\text { LAPLAND LONGSPUR } \\
\text { SNOWBUNTING } \\
\text { RUSTY BLACKBIRD } \\
\text { PINE GROSBEAK }\end{array}$ & $\begin{array}{l}20 \\
42\end{array}$ & & 30 & 2 & 2 & & & 1 & $\begin{array}{r}1546 \\
6 \\
\end{array}$ & $\begin{array}{r}25 \\
+ \\
16 \\
\end{array}$ & 2 \\
\hline $\begin{array}{l}\text { COMMION REDPOLL } \\
\text { HOARY REDPOLL } \\
\text { PINE SISKIN } \\
\text { EVENING GROSBEAK } \\
\text { HOUSE SPARROW }\end{array}$ & $\begin{array}{l}43 \\
41 \\
\end{array}$ & $\begin{array}{r}4 \\
20 \\
\end{array}$ & 30 & 16 & 235 & 122 & 15 & 51 & $\begin{array}{r}118 \\
1 \\
5 \\
248 \\
\end{array}$ & $\begin{array}{r}10 \\
20 \\
5 \\
45 \\
30 \\
\end{array}$ & 12 \\
\hline $\begin{array}{l}\text { NO. INDN. TABLES } 384 \\
\text { NO. SPECIES COUNT DAY } \\
\text { NO. SPECIES COUNT PERIOD } \\
\text { NO. SPECIES TABLES } 384 \\
\text { NO. INDIV. COUNT DAY } \\
\end{array}$ & \begin{tabular}{r|}
0 \\
14 \\
14 \\
0 \\
225 \\
\end{tabular} & \begin{tabular}{r|}
0 \\
7 \\
7 \\
0 \\
65
\end{tabular} & \begin{tabular}{r|}
0 \\
9 \\
11 \\
0 \\
88
\end{tabular} & \begin{tabular}{r|}
0 \\
8 \\
9 \\
0 \\
47 \\
\end{tabular} & $\begin{array}{r}0 \\
15 \\
18 \\
0 \\
359\end{array}$ & \begin{tabular}{r|}
1 \\
19 \\
19 \\
1 \\
308
\end{tabular} & $\begin{array}{r}0 \\
6 \\
6 \\
0 \\
162\end{array}$ & $\begin{array}{r}0 \\
13 \\
13 \\
0 \\
122 \\
\end{array}$ & $\begin{array}{r}7 \\
25 \\
25 \\
3 \\
2512\end{array}$ & $\begin{array}{r}0 \\
15 \\
18 \\
0 \\
237 \\
\end{array}$ & $\begin{array}{r}0 \\
10 \\
10 \\
0 \\
38\end{array}$ \\
\hline
\end{tabular}


Table 3-6. SPECIES RECORDED FROM FIVE OR MORE LOCALITIES $(+-$ a species seen during the count period but not on count day)

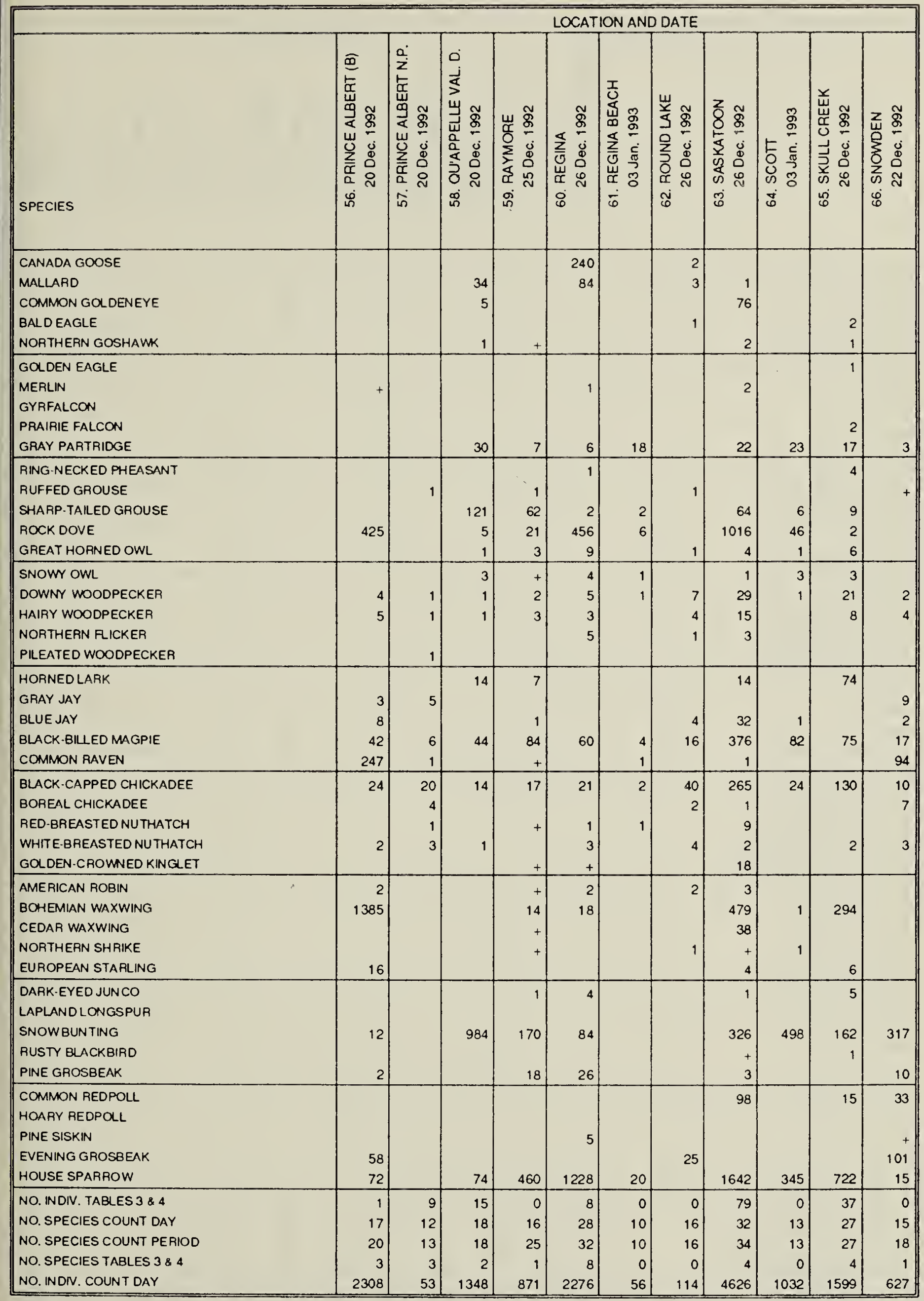


Table 3-7. SPECIES RECORDED FROM FIVE OR MORE LOCALITIES ( $+=$ a species seen during the count period but not on count day)

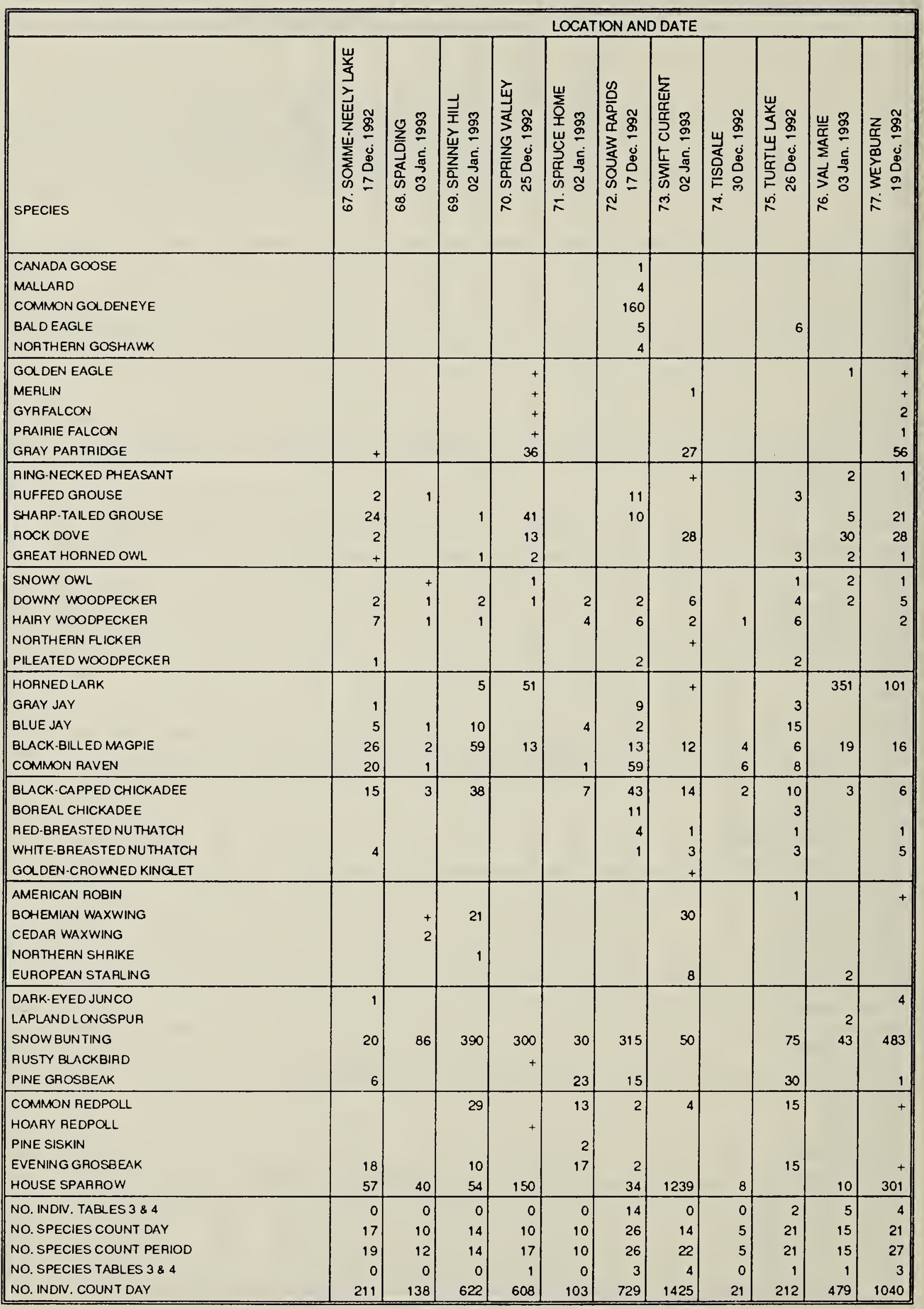


Table 3-8. SPECIES RECORDED FROM FIVE OR MORE LOCALITIES

$(+=$ a species seen during the count period but not on count day)

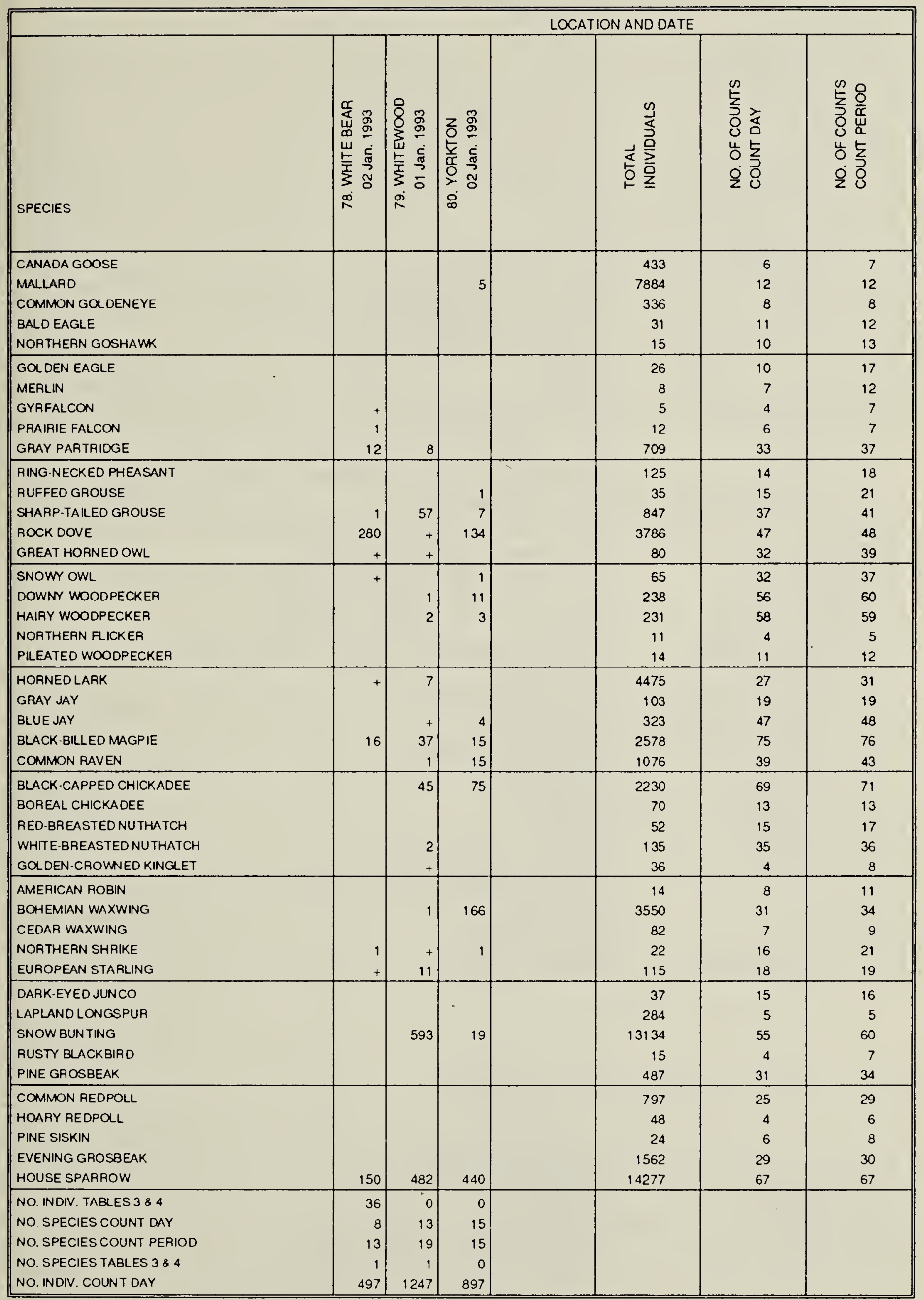


Table 4. SPECIES SEEN ON FEWER THAN FIVE COUNTS

\begin{tabular}{|c|c|}
\hline SPECIES & LOCATION AND NUMBER \\
\hline YELLOW-BILLED LOON & Gardiner Dam, 1 \\
\hline GREAT BLUE HERON & Crooked Lake, + \\
\hline TUNDRA SWAN & Fort Qu'Appelle, + \\
\hline AMERICAN BLACK DUCK & Regina, 2 \\
\hline CANVASBACK & Gardiner Dam, 1 \\
\hline LESSER SCAUP & Estevan, 7: Font Qu'Appelle, 1; Saskatoon, 1 \\
\hline BUFFLEHEAD & Estevan, 6; Fort Qu'Appelle, 1 \\
\hline COMMON MERGANSER & Gardiner Dam, 256; Squaw Rapids, 11 \\
\hline RED-BREASTED MERGANSER & Squaw Rapids, 1 \\
\hline RUDDY DUCK & Estevan, 1 \\
\hline NORTHERN HARRIER & Govenlock, 1 \\
\hline ROUGH-LEGGED HAWK & Biggar, 1: Govenlock, 6 \\
\hline SPRUCE GROUSE & Armit, 1; Prince Albert National Park, 2; Turtle Lake, 2 \\
\hline SAGE GROUSE & Grasslands National Park, 11 \\
\hline WILD TURKEY & Fort Qu'Appelle, + ; Fort Walsh, 4; White Bear, 36 \\
\hline AMERICAN COOT & Estevan, 14 \\
\hline MOURNING DOVE & Hudson Bay, + \\
\hline NORTHERN HAWK-OWL & Leader, 1 ; Prince Albert (B). + \\
\hline GREAT GRAY OWL & Armit, 1: Candle Lake, 3; Prince Albert National Park, + ; Snowden, + \\
\hline SHORT-EARED OWL & Big Muddy Lake, 3; Regina, + ; Skull Creok, 1 ; Spring Valley, +; Val Marie, 5 \\
\hline THREE-TOED WOODPECKER & Armit, 1: Candle Lake, 2; Regina,+ \\
\hline BLACK-BACKED WOODPECKER & Armit, 3; Kamsack, 1; MacDowall, 1; Squaw Rapids, 2 \\
\hline AMERICAN CROW & Moose Mountain, 1; Regina, 1: Skull Creok, 1 \\
\hline BROWN CREE PER & Fort Qu'Appelle, 1; Pike Lake, 1: Regina, 1: Saskatoon. 1: Switt Current, + \\
\hline TOWNSEND'S SOLITAIRE & Qu'Appelle Valley Dam, 1: Regina, + ; Switt Current + \\
\hline VARIED THRUSH & Swift Current, + \\
\hline AMERICAN TREE SPARROW & Fort Walsh, 15; Govenlock, 1: Grasslands National Park, 2: Raymore + \\
\hline LINCOLN'S SPARROW & Whit ewood, + \\
\hline WHITE-THROATED SPARROW & Prince Albert (B), + : S wift Current, + \\
\hline WHITE-CROWNED SPARROW & Pike Lake, 1 \\
\hline HARRIS' SPARROW & Prince Albert (B), 1: Skull Creek, 15 \\
\hline RED-WINGED BLACKBIRD & For Walsh, 4 \\
\hline WESTERN MEADOWLARK & Govenlock, 1 \\
\hline BREWER'S BLACKBIRD & Weyburn, 3 \\
\hline COMMON GRACKLE & Endeavour, 1 ; Regina, 2; Weyburn, 1 \\
\hline ROSY FINCH & Fort Walsh, 23, Grasslands National Park, 6. Skull Creck, 20 \\
\hline PURPLE FINCH & Crooked Lake $\theta_{1}$ \\
\hline HOUSE FINCH & Reyina, 2; Weyburn, + \\
\hline WHITE-WINGED CROSSBILL & Marsden, 5; Pike Lake, 5 \\
\hline
\end{tabular}

Table 5. BIRDS NOT IDENTIFIED TO SPECIES

\begin{tabular}{|l|l|}
\hline SPECIES & LOCATI ON AND NUMBER \\
\hline UNIDENTIFIED BIRD & Cabri, 1; Prince Albert National Park, 7 \\
\hline WOODPECKER species & Big Muddy Lake, 1 \\
\hline WAXWING species & Qu'Appelle Valley Dam, 14: Saskatoon, 25 \\
\hline REDPOLL species & Big Muddy Lake, 6; For Walsh, 40; Saskatoon, 52 \\
\hline
\end{tabular}


Table 6. SUMMARY OF NEW OR TYING HIGH COUNTS ESTABLISHED DURING THE 1992 COUNTS (tying counts regular type; boldface/italic indicates a new record;

$\mathrm{CP}=$ count period species)

\begin{tabular}{||l|c|l|c|l||}
\hline LOCATION & $\begin{array}{c}1992 \\
\text { COUNT }\end{array}$ & SPECIES & $\begin{array}{c}\text { PREVIOUS } \\
\text { HIGH }\end{array}$ & LOCATION AND YEAR \\
\hline Gardiner Dam & 1 & Yellow-billed Loon & 1 & Gardiner Dam (91) \\
\hline [Crooked Lake CP] & 1 & Great Blue Heron & 1 & Barrier Lake (88); Fort Walsh (85) \\
\hline Regina & 2 & American Black Duck & 1 & north to Gardiner Dam \\
\hline Estevan & 6 & Bufflehead & 4 & Douglas Provinclal Park (88) \\
\hline Govenlock & 1 & Northern Harrier & 1 & north to Harris \\
\hline Govenlock & 5 & Pralie Falcon & 4 & Govenlock (86) \\
\hline While Bear & 36 & Wild Turkey & 26 & Fort Walsh (88) \\
\hline Estevan & 14 & American Coot & 11 & Regina (63) \\
\hline Kamsack & 15 & White-breasted Nuthatch & 15 & Round Lake (84) \\
\hline [Swift Current CP] & 1 & Varied Thrush & 1 & north to Pierce Lake \\
\hline [Whitewood CP] & 1 & Lincoln's Sparrow & & NEW \\
\hline Pike Lake & 1 & White-crowned Sparrow & 1 & north to Saskatoon \\
\hline Skull Creek & 15 & Harris' Sparrow & 3 & Govenlock (86) \\
\hline Regina and [Weyburn CP] & 2 & House Flnch & & NEW \\
\hline Kam sack & 564 & Evening Grosbeak & 515 & Kam sack (85) \\
\hline
\end{tabular}

Table 7. COMPARISON OF 1992 POPULATIONS TO 1991 AND AVERAGE PAST YEARS (1987-1991) BASED ON PARTY HOURS PER INDIVIDUAL

\begin{tabular}{|c|c|c|c|c|c|}
\hline SPECIES & 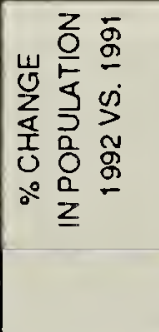 & 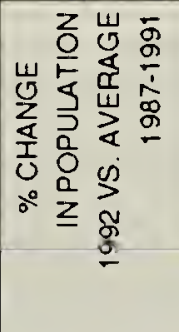 & SPECIES & 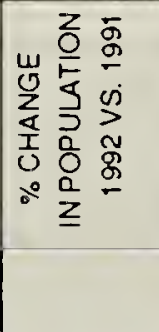 & 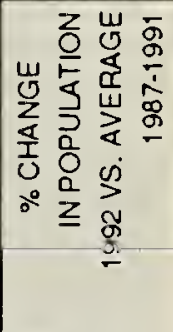 \\
\hline CANADA GOOSE & 16.3 & -65.1 & COMMON RAVEN & -14.3 & 13.4 \\
\hline MALLARD & 217.0 & 21.0 & BLACK-CAPPED CHICKADEE & 7.5 & 23.2 \\
\hline COMMON GOLDENEYE & 102.4 & -15.5 & BOREAL CHICKADEE & 34.6 & -2.8 \\
\hline BALD EAGLE & 106.7 & 10.7 & RED-BREASTED NUTHATCH & -67.9 & -54.8 \\
\hline NORTHERN GOSHAWK & -16.7 & 0.0 & WHITE-BREASTED NUTHATCH & 23.9 & 50.0 \\
\hline GOLDEN EAGLE & -35.0 & -33.3 & BROWN CREEPER & 0.0 & -20.0 \\
\hline MERLIN & 14.3 & -52.9 & GOLDEN-CROWNED KINGLET & 125.0 & 16.1 \\
\hline PRAIRIE FALCON & 71.4 & 140.0 & AMERICAN ROBIN & -26.3 & -30.0 \\
\hline GRAY PARTRIDGE & -57.5 & -48.3 & BOHEMIAN WAXWING & -12.1 & -56.7 \\
\hline RING-NECKED PHEASANT & 10.6 & -16.7 & CEDAR WAXWING & .45 .0 & .38 .3 \\
\hline SPRUCE GROUSE & -64.3 & -44.4 & NORTHERN SHRIKE & -35.3 & 4.8 \\
\hline RUFFED GROUSE & -61.5 & -61.1 & EUROPEAN STARLING & -42.2 & -55.9 \\
\hline SHARP-TAILED GROUSE & -37.7 & -16.5 & AMERICAN TREE SPARROW & -35.7 & -57.1 \\
\hline ROCK DOVE & -20.7 & -30.3 & DARK-EYED JUNCO & -62.6 & -27.5 \\
\hline GREAT HORNED OWL & 3.9 & -12.1 & LAPLAND LONGSPUR & -75.3 & -88.5 \\
\hline SNOWY OWL & 27.5 & 20.4 & SNOW BUNTING & 24.4 & -19.0 \\
\hline SHORT-EARED OWL & 125.0 & -47.1 & RED-WINGED BLACKEIRD & -89.7 & -69.2 \\
\hline DOWNY WOODPECKER & -2.4 & 18.9 & RUSTY ELACKBIRD & -60.5 & -59.5 \\
\hline HAIRY WOODPECKER & 3.1 & 17.8 & PINE GROSBEAK & -60.8 & -35.1 \\
\hline THREE-TOED WOOOPECKER & -57.1 & -62.5 & RED CROSSBILL & -100.0 & -100.0 \\
\hline NORTHERN FLICKER & -57.7 & .31 .3 & WHITE-WINGED CROSSBILL & -97.2 & -95.7 \\
\hline PILEATED WOODPECKER & -41.7 & -12.5 & COMMON REDPOLL & -85.3 & -76.9 \\
\hline HORNED LARK & 349.8 & 40.4 & HOARY REDPOLL & -72.4 & -61.9 \\
\hline GRAY JAY & -9.6 & 13.2 & PINE SISKIN & .72 .7 & -60.0 \\
\hline BLUE JAY & -36.7 & -1.8 & EVENING GROSBEAK & -9.3 & 4.5 \\
\hline BLACK-BILLED MAGPIE & -14.0 & 0.6 & HOUSE SPARROW & -8.8 & -14.8 \\
\hline
\end{tabular}

